\title{
NUMBER OF SOLUTIONS WITH A NORM BOUNDED BY A GIVEN CONSTANT OF A SEMILINEAR ELLIPTIC PDE WITH A GENERIC RIGHT-HAND SIDE
}

\author{
ALEXANDER NABUTOVSKY
}

\begin{abstract}
We consider a semilinear boundary value problem $-\Delta u+f(u, x)=$ 0 in $\Omega \subset \mathbb{R}^{N}$ and $u=0$ on $\partial \Omega$. We assume that $f$ is a $C^{\infty}$-smooth function and $\Omega$ is a bounded domain with a smooth boundary. For any $C^{\alpha}-$ smooth perturbation $h(x)$ of the right-hand side of the equation we consider the function $N_{h}(S)$ defined as the number of $C^{2+\alpha}$-smooth solutions $u$ such that $\|u\|_{C^{0}(\Omega)} \leq S$ of the perturbed problem.

How "small" $N_{h}(S)$ can be made by a perturbation $h(x)$ such that $\|h\|_{C^{0}(\Omega)}$ $\leq \varepsilon$ ? We present here an explicit upper bound in terms of $\varepsilon, S$ and

$$
\max _{|u| \leq S, x \in \bar{\Omega}}\left\|D_{u}^{i} f(u, x)\right\| \quad(i \in\{0,1,2\}) .
$$

If $S$ is fixed then $h$ can be chosen by such a way that the upper bound persists under small in $C^{0}$-topology perturbations of $h$. We present an explicit lower bound for the radius of the ball of such admissible perturbations.
\end{abstract}

\section{INTRODUCTION}

Let an elliptic problem

$$
\begin{cases}-\Delta u+f(u, x)=0 & \text { in } \Omega, \\ u=0 & \text { on } \partial \Omega,\end{cases}
$$

be given. We assume that $\Omega \subset \mathbb{R}^{N}$ is a bounded domain with a $C^{\infty}$-smooth boundary and $f: \mathbb{R} \times \bar{\Omega} \rightarrow \mathbb{R}$ is a $C^{\infty}$-smooth function. We consider this problem in the space $C^{2+\alpha}(\bar{\Omega})$ for some $\alpha \in(0,1)$.

The set of solutions of the problem (1.1) can be uncountable. However, it follows from Smale results that there exists an arbitrarily small $C^{\alpha}$-smooth perturbation of the right-hand side of (1.1a) making the solution set of the problem (1.1) discrete. More precisely it follows from results of [13] that for any $\varepsilon>0$ there exists a function $h \in C^{\alpha}(\bar{\Omega})$ such that

$$
\max _{x \in \Omega}|h(x)| \leq \varepsilon .
$$

(2) All solutions of the problem

$$
\left\{\begin{array}{l}
-\Delta u+f(u, x)=h(x) \text { in } \Omega \\
\left.u\right|_{\partial \Omega}=0
\end{array}\right.
$$

are isolated.

Received by the editors March 23, 1990.

1980 Mathematics Subject Classification (1985 Revision). Primary 35J65, 58G20, 34B15; Secondary $58 \mathrm{C} 25$. 
The solution set of the problem (1.3) can be characterized by the function $N_{h}(S)$ defined as the number of $C^{2+\alpha}$-smooth solutions of (1.3) such that $\|u\|_{C^{0}(\bar{\Omega})} \leq S$. We are interested in the following informal question: How "small" can $N_{h}(S)$ be made by a $C^{\alpha}$-smooth perturbation $h$ of the right-hand side of (1.1a), such that $\|h\|_{C^{0}(\bar{\Omega})} \leq \varepsilon$ ?

Ehrmann [4] and Fučik and Lovicar [7] (see also [8]) have proved that the ordinary differential equation

$$
\left\{\begin{array}{l}
u^{\prime \prime}+f(u)=h(x), \quad x \in(0, \pi), \\
u(0)=u(\pi)=0,
\end{array}\right.
$$

has an infinite number of classical solutions $u$ for any continuous right-hand side $h$ when $f$ is a continuous function and

$$
\lim _{t \rightarrow \pm \infty} \frac{f(t)}{t}=+\infty
$$

Other results concerning the existence of infinitely or arbitrarily many solutions of semilinear elliptic equations can be found in $[1,11,24]$ and in the survey [23] (see also references there).

These results imply that in a general case we cannot expect to make $N_{h}(S)$ bounded for all $S$ by any $C^{\alpha}$-smooth perturbation $h$. They also suggest that the forementioned question can be posed either concerning a value $N_{h}(S)$ for some fixed $S$ or concerning the asymptotic growth of $N_{h}(S)$ when $S \rightarrow \infty$.

Below we give a partial answer for these questions. Namely, we present explicitly depending on $S$, $\varepsilon, h_{0}$, and $f$ upper bounds on $N_{h}(S)$ for a properly chosen $h \in C^{\alpha}(\bar{\Omega})$ such that $\left\|h-h_{0}\right\|_{C^{0}(\bar{\Omega})} \leq \varepsilon$. If $S$ is fixed then $h$ can be chosen by such a way that our estimates of $N_{h}(S)$ persist under perturbations of $h$ which are small in $C^{0}$-topology. We present also a lower bound for the radius of the ball of such admissible $h$.

Now we can state our main results. We will denote the diameter of $\Omega$ in the metric of $\mathbb{R}^{N}$ by $\operatorname{diam} \Omega$ and

$$
\sup _{x_{1}, x_{2} \in \Omega} \frac{\inf _{\omega \in C^{1}([0,1], \bar{\Omega}) ; \omega(0)=x_{1} ; \omega(1)=x_{2}} \text { length }(\omega)}{\left|x_{1}-x_{2}\right|}
$$

by dil $\Omega$. Let $M_{0}(S)$ denote $\max _{|u| \leq S, x \in \bar{\Omega}}|f(u, x)|$ and $M_{i}(S)$ denote $\max _{|u| \leq S, x \in \bar{\Omega}}\left\|D_{u}^{i} f(u, x)\right\| \quad(i \in\{1,2\})$. Further, let

$$
\bar{M}_{0}(S)=\max \left\{1, M_{0}(S)\right\}, \quad \bar{M}_{2}(S)=\max \left\{1, M_{1}(S), M_{2}(S)\right\} .
$$

Theorem 1.1. Let $\Omega \subset \mathbb{R}^{N}$ be a bounded domain with a $C^{\infty}$-smooth boundary and $f \in C^{\infty}(\mathbb{R} \times \bar{\Omega})$ be an arbitrary function such that for any constant $c>0$, $\bar{M}_{2}\left(S+c / \bar{M}_{2}(S)\right)=\bar{M}_{2}(S)(1+o(1))$ when $S \rightarrow \infty$.

There exist functions $\Theta_{1}(N, \operatorname{diam} \Omega), \Theta_{2}(N, \operatorname{diam} \Omega)$ and a function $\nu(S, \varepsilon)$ with the following property. Let $S>0$ and $\alpha \in(0,1)$ be fixed. For any $h_{0} \in C^{\alpha}(\bar{\Omega})$ and positive $\varepsilon<1$ there exists $h \in C^{\alpha}(\bar{\Omega})$ such that

(1) $h-h_{0} \in C^{2+\alpha}(\bar{\Omega})$ and $\left\|h-h_{0}\right\|_{C^{1}(\bar{\Omega})} \leq \varepsilon$. 
(2) For any $g \in C^{\alpha}(\bar{\Omega})$ such that $\|g-h\|_{C^{0}(\bar{\Omega})} \leq \nu(S, \varepsilon)$, the number $N_{g}(S)$ of $C^{2+\alpha}$-smooth solutions of the boundary value problem

$$
\left\{\begin{array}{l}
-\Delta u+f(u, x)=g(x) \text { in } \Omega, \\
\left.u\right|_{\partial \Omega}=0,
\end{array}\right.
$$

such that $\|u\|_{C^{0}(\bar{\Omega})} \leq S$ satisfies the inequality

$$
\begin{aligned}
\ln N_{g}(S) \leq \Theta_{1}(N, \operatorname{diam} \Omega)( & (\operatorname{dil} \Omega)^{N} S^{N}\left(\bar{M}_{0}(S)+\left\|h_{0}\right\|_{C^{0}(\bar{\Omega})}\right)^{N} \\
& \left.\times\left(\bar{M}_{2}(S)\right)^{7 N^{2} / 4+N}+\bar{M}_{2}(S)^{N} \ln \frac{1}{\varepsilon}\right) .
\end{aligned}
$$

(3) The function $\nu(S, \varepsilon)$ satisfies the inequality

$$
-\ln \nu(S, \varepsilon) \leq \Theta_{2}(N, \operatorname{diam} \Omega)\left((\operatorname{dil} \Omega)^{N} S^{N}\left(\bar{M}_{0}(S)+\left\|h_{0}\right\|_{C^{0}(\bar{\Omega})}\right)^{N}\right.
$$

$$
\left.\times\left(\bar{M}_{2}(S)\right)^{7 N^{2} / 4+N / 2}+\bar{M}_{2}(S)^{N / 2} \ln \frac{1}{\varepsilon}\right) .
$$

Theorem 1.2. Let $\Omega \subset \mathbb{R}^{N}$ be a bounded domain with a $C^{\infty}$-smooth boundary and $f \in C^{\infty}(\mathbb{R} \times \bar{\Omega})$ be an arbitrary function such that

$$
\lim _{S \rightarrow+\infty} \bar{M}_{2}(S)=+\infty \quad \text { and } \lim _{S \rightarrow+\infty} \frac{\bar{M}_{2}\left(S+c / \bar{M}_{2}(S)\right)}{\bar{M}_{2}(S)}=1 .
$$

Let $\delta>0$ and $\alpha \in(0,1)$ be fixed.

There exists an increasing sequence $\left\{S_{j}\right\}_{j=1}^{\infty}$ tending to infinity and a function $\Theta(N$, diam $\Omega)$ with the following property. For any $h_{0} \in C^{\alpha}(\bar{\Omega})$ and any positive $\varepsilon \leq \frac{1}{2}$ there exists $h \in C^{\alpha}(\bar{\Omega})$ such that

(1) $h-h_{0} \in C^{1}(\bar{\Omega})$ and $\left\|h-h_{0}\right\|_{C^{1}(\bar{\Omega})} \leq \varepsilon$.

(2) For any $j \geq 1$ the number $N_{h}\left(S_{j}\right)$ of $C^{2+\alpha}$-smooth solutions of the boundary value problem

$$
\left\{\begin{array}{l}
-\Delta u+f(u, x)=h(x) \text { in } \Omega, \\
\left.u\right|_{\partial \Omega}=0,
\end{array}\right.
$$

such that $\|u\|_{C^{0}(\bar{\Omega})} \leq S_{j}$ satisfies the following inequality

$$
\begin{aligned}
\ln N_{h}\left(S_{j}\right) \leq \Theta(N, \operatorname{diam} \Omega)[ & (\operatorname{dil} \Omega)^{N} S_{j}^{N+\delta}\left(\bar{M}_{0}\left(S_{j}\right)+\left\|h_{0}\right\|_{C^{0}(\bar{\Omega})}\right)^{N} \\
& \left.\times \bar{M}_{2}\left(S_{j}\right)^{7 N^{2} / 4+N}+\bar{M}_{2}\left(S_{j}\right)^{N+\delta} \ln \frac{1}{\varepsilon}\right] .
\end{aligned}
$$

Remark 1. Theorems 1.1 and 1.2 can be proven without the assumption that for any $c>0, \bar{M}_{2}\left(S+c / \bar{M}_{2}(S)\right)=\bar{M}_{2}(S)(1+o(1))$ when $S \rightarrow \infty$. But proofs will be more awkward without this assumption.

Remark 2. It will be seen from the proof that the numbers $S_{i}$ mentioned in Theorem 1.2 can be effectively majorized. So, because of the monotonicity 
of $N_{h}(S)$ we also have an estimate on $\ln N_{h}(S)$ for all $S$ and not only on $\ln N_{h}\left(S_{i}\right)$.

Remark 3. The proofs of Theorems 1.1, 1.2 are constructive. They allow one to find explicit expressions for the functions $\Theta_{1}(N, \operatorname{diam} \Omega), \Theta_{2}(N$, diam $\Omega)$, and $\Theta(N, \operatorname{diam} \Omega)$ and, thus, to make estimates (1.7), (1.8), and (1.9) completely explicit.

Remark 4. Let $\mathscr{H}\left(h_{0}, \varepsilon\right)$ denote the set of $h$ satisfying the statements (1)-(3) of Theorem 1.1 for some fixed $h_{0}, \varepsilon$. Let $\mathscr{H}=\bigcup_{\varepsilon, h_{0}} \mathscr{H}\left(h_{0}, \varepsilon\right)$. It follows from Theorem 1.1 that $\mathscr{H}$ will be an open dense subset of the space of all $C^{\alpha}$-smooth functions on $\bar{\Omega}$ equipped with the $C^{0}$-topology.

Similarly, let $\mathscr{H}\left(h_{0}, \varepsilon,\left\{S_{j}\right\}\right)$ denote the set of $C^{\alpha}$-smooth right-hand sides $h(x)$ of $(1.3 \mathrm{a})$ such that the statement of Theorem 1.2 holds for them at some fixed $h_{0}, \varepsilon,\left\{S_{j}\right\}$. Let

$$
\widetilde{\mathscr{H}}=\bigcup_{\substack{\varepsilon>0, h_{0} \\\left\{S_{j}\right\}}} \mathscr{H}\left(h_{0}, \varepsilon,\left\{S_{j}\right\}\right) .
$$

Then $\widetilde{\mathscr{H}}$ coincides with the set of all $C^{\alpha}$-smooth right-hand sides of (1.3a) for which the solution set is discrete. Thus, by the Smale-Sard Theorem [13] $\widetilde{\mathscr{H}}$ contains a dense $G_{\delta}$ subset of the space of all $C^{\alpha}$-smooth functions on $\bar{\Omega}$ equipped with the $C^{0}$-topology.

This remark explains the word "generic" in the title of the paper.

The method which we use to prove the theorem can briefly be described as follows:

Denote the nonlinear operator in the left-hand side of (1.1a) by $F(u)$. Its derivative at a given point $u_{0}$ can be expressed as

$$
F^{\prime}\left(u_{0}\right) v=-\Delta v+f_{u}^{\prime}\left(u_{0}, x\right) v .
$$

Let $u_{0}$ be fixed and some positive reals $a, b$ be given. The space $C^{2+\alpha}(\bar{\Omega})$ can be decomposed in a direct sum $P_{1} \oplus P_{2}$, where $P_{1}$ is a finite-dimensional space of a known dimension and $P_{2}$ has the following property. Let $v \in P_{2}$, and $\left.v\right|_{\partial \Omega}=0$, and $\left\|u-u_{0}\right\|_{L^{2}(\bar{\Omega})} \leq b / M_{2}(S)$. Then

$$
\left\|\left.F^{\prime}(u)\right|_{P_{2}} v\right\|_{L^{2}(\bar{\Omega})} \geq a\|v\|_{L^{2}(\bar{\Omega})} .
$$

Using the implicit function theorem we establish a correspondence between solutions of (1.3) close enough to $u_{0}$ (in $C^{0}$ norm) and solutions of a certain nonlinear finite-dimensional equation $T_{h}(x)=0$ in a ball in $P_{1}$. However to apply the implicit function theorem we need in particular an explicit upper estimate of the norm of the operator $\left[F^{\prime}\left(u_{0}\right) \mid P_{2}\right]^{-1}: \operatorname{Im} F^{\prime}\left(u_{0}\right) \mid P_{2} \rightarrow P_{2}$, where $P_{2}$ and $\left.\operatorname{Im} F^{\prime}\left(u_{0}\right)\right|_{P_{2}}$ are equipped with $C^{0}$-topology. This operator is bounded due to the ellipticity of $F^{\prime}\left(u_{0}\right)$. An explicit estimate of its norm can be deduced using the standard techniques of the theory of elliptic PDE's.

Let $\|u\|_{C^{0}(\bar{\Omega})} \leq S$ and $u$ be a solution of (1.3). Then estimates on $\|u\|_{C^{1}(\bar{\Omega})}$ can also be derived. Thus, a set of all solutions of problems (1.3) for all $h$ satisfying (1.2) belongs to a compact subset of $C^{0}(\bar{\Omega})$. This set can be covered by a finite number of balls in which we can reduce the problem to a finitedimensional case. Using such a cover it is possible to reduce a search for the 
perturbation, existence of which is stated in Theorem 1.1, to a search in a finitedimensional set for a point such that its projections on certain linear subspaces will not be near-critical values of the finite-dimensional mappings corresponding to the unperturbed problem (1.1) in neighborhoods of the centres of balls of the cover.

To prove the existence of such points we use the result of Yomdin (a quantitative version of the classic Sard's theorem) $[14,15]$ which gives an estimate on the number of balls of a given radius covering a set of near-critical values of a smooth finite-dimensional mapping. It should be noted that a number of authors used the Smale-Sard theorem + the existence of a priori bounds in order to prove the finiteness of the solution set of a generic nonlinear boundary value problem (cf. [5, 6, 12]). However, their results are not constructive because the classic finite-dimensional Sard's theorem is the pure existence theorem.

The proof of Theorem 1.2 is somewhat more intricate. The required perturbation of $h_{0}$ is constructed inductively. At each step a perturbation $h_{i}$ is constructed as in the proof of Theorem 1.1. It makes $N_{\Sigma_{j=0}^{i} h_{j}}\left(S_{i}\right)$ satisfy the inequality (1.7) for $S_{i}$ as in the proof of Theorem 1.1. Moreover, this can be done in such a way that $N_{h}\left(S_{i}\right)$ for all $h$ close enough to $\sum_{j=0}^{i} h_{j}$ would also satisfy (1.7). So $\sum_{j=0}^{i} h_{j}$ may be perturbed further to make $N_{\Sigma_{j=0}^{i+1} h_{j}}\left(S_{i+1}\right)$ satisfy the inequality $(1.7)$, etc.

It may occur that for boundary value problems for ordinary differential equations the estimates given by (1.7), (1.9) can be significantly improved. We conjecture that in the case $N=1$ it is possible to prove upper bounds on $N_{g}(S)$ in Theorems 1.1 and 1.2 of the form

$$
N_{g}(S) \leq \Theta(\text { length } \Omega)\left(S M_{2}(S)\right)^{\alpha},
$$

where $\alpha$ is a constant.

However, we believe that for the case of arbitrary $N$ the upper bounds on $N_{g}(S)$ in Theorem 1.1 cannot be made polynomially dependent on $\left\|h_{0}\right\|_{C^{0}(\bar{\Omega})}$, $M_{0}(S), M_{1}(S), M_{2}(S)$. The reason is that the dimension of the kernel of the linearized operator can depend on $M_{1}(S)$ polynomially and that the cardinality of the inverse image set of a generic value of a quadratic mapping of the unit ball in $\mathbb{R}^{m}$ into itself can be $2^{m}$.

It is obvious from the proof that the methods and results of this paper can be easily generalized for a general class of elliptic operators and boundary conditions.

Another possible way to generalize Theorems 1.1 and 1.2 is to consider a nonlinear boundary value problem

$$
\begin{cases}-\Delta u+f(u, x, \lambda)=0 & \text { in } \Omega, \\ u=0 & \text { on } \partial \Omega,\end{cases}
$$

smoothly depending on a parameter $\lambda$. (In the situation of Theorems 1.1 and 1.2 the right-hand side $h$ can be considered as this parameter.) The analogs of Theorems 1.1 and 1.2 will state that in the $\varepsilon$-neighborhood of any $\lambda_{0}$ there exists $\lambda$ such that the number of solutions of (1.12) satisfies some prescribed in advance estimates, similar to (1.7) or (1.9).

This work can be regarded as a work on geometry of nonlinear elliptic operators on Banach spaces. In this connection, we would like to mention papers 
[17-20, 22]. Although results of these papers are not related directly with our results, geometric approaches were used there in order to investigate properties of specific semilinear operators in Banach spaces and, in particular, to obtain results about the multiplicity of solutions of corresponding boundary value problems when the right-hand side of the equation varies.

\section{SOME EXPLICIT A PRIORI ESTIMATES FOR SOLUTIONS OF SEMILINEAR ELLIPTIC EQUATIONS}

Let $P_{1}$ be a finite-dimensional space spanned by eigenfunctions of a Schrödinger operator $-\Delta+\varphi(x)$ in $L_{0}^{2}(\bar{\Omega})$ corresponding to its eigenvalues $\lambda \leq \lambda_{0}$ where $\lambda_{0}$ is a given constant. If $\varphi(x) \in C^{2+\alpha}(\bar{\Omega})$, then $P_{1} \subset C^{2+\alpha}(\bar{\Omega}) \subset C^{1}(\bar{\Omega})$. Thus, we can consider norms \|\|$_{L^{2}}$ and \|\|$_{C^{1}}$ on $P_{1}$ inherited from $L^{2}(\bar{\Omega})$ and $C^{1}(\bar{\Omega})$, correspondingly. Obviously, $\|u\|_{L^{2}} \leq \sqrt{|\Omega|}\|u\|_{C^{0}}$. Because $P_{1}$ is finite dimensional there exists an estimate of the type $\|u\|_{C^{1}} \leq \tau\left(\varphi, \lambda_{0}\right)\|u\|_{L^{2}}$, for all $u \in P_{1}$. In this section we present an explicit expression for $\tau\left(\varphi, \lambda_{0}\right)$ (Proposition 2.3).

The equations (1.3) together with the inequality (1.2) and the assumption $\|u\|_{C^{0}(\bar{\Omega})} \leq S$ imply the existence of an a priori bound on $\|u\|_{C^{1}(\bar{\Omega})}$. An explicit upper bound on $\|u\|_{C^{1}(\bar{\Omega})}$ is given by Proposition 2.2. Hence the set of solutions $u$ such that $\|u\|_{C^{0}(\bar{\Omega})} \leq S$ of all boundary value problems (1.3) with $h$ satisfying (1.2) is compact. Thus, it can be covered by a finite number of $C^{0}$-balls of a fixed radius. Proposition 2.4 estimates a number of balls of prescribed radii necessary to cover this solution set.

To prove the inequality $\|u\|_{C^{1}(\bar{\Omega})} \leq \tau\left(\varphi, \lambda_{0}\right)\|u\|_{L^{2}(\bar{\Omega})}$ for $u \in P_{1}$ mentioned before, we prove the following proposition, which also will be used to estimate the $C^{0}$-norm of a solution $v$ of a nonhomogeneous boundary value problem

$$
\left\{\begin{array}{l}
-\Delta v(x)+\varphi(x) v(x)=h(x) \quad \text { in } \Omega \\
\left.v\right|_{\partial \Omega}=0
\end{array}\right.
$$

in terms of $L^{2}$-norm of $v$ and $C^{0}$-norm of $h$. We prove this proposition using well-known techniques introduced by De Giorgi. Some results similar to the result of this proposition and other methods of proving results of such type can be found in [3, Appendices 5-7] (see also references there).

Proposition 2.1. Let $v \in C_{0}^{2}(\bar{\Omega})$ (i.e., $\left.v\right|_{\partial \Omega}=0$ ) and $|\Delta v(x)| \leq A|v(x)|+B$ for some $A>0, B \geq 0$ and for all $x \in \bar{\Omega}$. Then

$$
\|v\|_{C^{0}(\bar{\Omega})} \leq \max \left\{\frac{B}{A}, C(N) \sqrt{|\Omega|} A^{N / 2}\|v\|_{L^{2}(\bar{\Omega})}\right\},
$$

where

$$
C(N)=\left\{\begin{array}{l}
\frac{3 \sqrt{3}}{2 \pi} \text { for } N=1, \\
\frac{1}{2}(N+2)^{N / 2+1} w_{N}^{-1} j_{N / 2-1}^{-N} \text { for } N \geq 2 .
\end{array}\right.
$$

Here $w_{N}=2 \pi^{N / 2} /(N \Gamma(N / 2))$ is the volume of the unit ball in $\mathbb{R}^{N}$ and $j_{N / 2-1}$ is the first zero of the Bessel function of order $(N / 2-1)$.

Remark. The value of $C(N)$ given by (2.2) can be significantly improved (for example, using a symmetrization). 
Proof. First, suppose that $v \in C_{0}^{\infty}(\bar{\Omega})$. Consider a component of the set $\{x \in$ $\Omega \mid v(x) \neq 0\}$. Denote it by $\Omega^{\prime}$. Without loss of generality we may assume that $v(x)>0$ for any $x \in \Omega^{\prime}$.

Let

$$
\begin{gathered}
D_{\beta}=\left\{x \in \Omega^{\prime} \mid v(x) \geq \beta\right\}, \quad \lambda(\beta)=\int_{D_{\beta}}(v(x)-\beta) d x, \\
\operatorname{meas}(\beta)=\text { the measure of } D_{\beta}, \quad S=\max _{x \in \Omega^{\prime}} v(x) .
\end{gathered}
$$

By the Cavalieri principle

$$
\lambda(\beta)=\int_{D_{\beta}}(v(x)-\beta) d x=\int_{\beta}^{S} \operatorname{meas}(t) d t .
$$

Hence

$$
\lambda^{\prime}(\beta)=-\operatorname{meas}(\beta) .
$$

Note that if $\beta$ is not a critical value of $v$ then $\partial D_{\beta}$ is smooth. By Sard's theorem the set of critical values of $v$ is a set of zero measure. Up to the formula $\left(2.6^{\prime \prime}\right)$ we will assume that $\beta$ is not a critical value of $v$.

By Schwarz's inequality

$$
\lambda(\beta) \leq\left[\int_{D_{\beta}}(v(x)-\beta)^{2} d x\right]^{1 / 2} \operatorname{meas}(\beta)^{1 / 2} .
$$

By the minimax property of eigenvalues

$$
\left[\int_{D_{\beta}}(v(x)-\beta)^{2} d x\right]^{1 / 2} \leq \frac{1}{\sqrt{\lambda_{\min }(\beta)}}\left[\int_{D_{\beta}} \operatorname{grad}^{2} v(x) d x\right]^{1 / 2}
$$

where $\lambda_{\min }(\beta)$ is the minimal eigenvalue of $-\Delta$ in $D_{\beta}$ with zero Dirichlet boundary conditions. By the Faber-Krahn inequality (cf. [2, Theorem 3.8])

$$
\lambda_{\min }(\beta) \geq \begin{cases}\pi^{2} /(\operatorname{meas}(\beta))^{2}, & N=1, \\ {\left[w_{N} / \operatorname{meas}(\beta)\right]^{2 / N} j_{N / 2-1}^{2},} & N \geq 2 .\end{cases}
$$

Applying Green's formula we get

$$
\begin{aligned}
& {\left[\int_{D_{\beta}} \operatorname{grad}^{2} v(x) d x\right]^{1 / 2}=\sqrt{\int_{D_{\beta}}-\Delta v(x)(v(x)-\beta) d x}} \\
& \quad \leq \sqrt{\int_{D_{\beta}}(A v(x)+B)(v(x)-\beta) d x} \leq(A S+B)^{1 / 2} \lambda^{1 / 2}(\beta) .
\end{aligned}
$$

Combining (2.6) and (2.6 ) with (2.5) and afterwards substituting the resulting inequality into (2.4) we get

$$
\lambda(\beta) \leq C_{1}^{2}(N)(A S+B) \operatorname{meas}(\beta)^{(N+2) / N},
$$

where

$$
C_{1}(N)= \begin{cases}\frac{1}{\pi}, & \text { for } N=1 \\ w_{N}^{-1 / N} j_{N / 2-1}^{-1}, & \text { for } N \geq 2\end{cases}
$$


Thus,

$$
-\lambda^{\prime}(\beta)=\operatorname{meas}(\beta) \geq\left[C_{1}^{2}(N)(A S+B)\right]^{-N /(N+2)} \lambda(\beta)^{N /(N+2)},
$$

i.e.,

$$
\lambda^{\prime}(\beta) \leq-\left[C_{1}^{2}(N)(A S+B)\right]^{-N /(N+2)} \lambda(\beta)^{N /(N+2)}
$$

if $\beta$ is not a critical value of $v$. So, $\left(2.6^{\prime \prime}\right)$ holds for almost all $\beta$. It follows from the monotonicity of $\lambda$ that there exists an inverse function $\beta(\lambda):[0, \lambda(0)]$ $\rightarrow[0, S]$. Rewriting $\left(2.6^{\prime \prime}\right)$ as the inequality on $\beta^{\prime}(\lambda)$ and integrating it we can prove that for any $\lambda \in[0, \lambda(0)]$,

$$
\beta(\lambda) \geq S-\left[C_{1}^{2}(N)(A S+B)\right]^{N /(N+2)} \frac{N+2}{2} \lambda^{2 /(N+2)} .
$$

Hence

$$
0=\beta(\lambda(0)) \geq S-\left[C_{1}^{2}(N)(A S+B)\right]^{N /(N+2)}\left(\frac{N}{2}+1\right) \lambda(0)^{2 /(N+2)} .
$$

It follows from (2.8) that

$$
\|v\|_{L^{1}\left(\Omega^{\prime}\right)} \equiv \lambda(0) \geq\left(\frac{N}{2}+1\right)^{-(N / 2+1)} C_{1}^{-N}(N)\left(A+\frac{B}{S}\right)^{-N / 2} S .
$$

But

$$
\|v\|_{L^{1}\left(\Omega^{\prime}\right)} \leq\|v\|_{L^{2}\left(\Omega^{\prime}\right)} \sqrt{\left|\Omega^{\prime}\right|} \leq\|v\|_{L^{2}(\bar{\Omega})} \sqrt{|\Omega|} .
$$

Combining (2.9) and (2.10) we get

$$
2^{-N / 2} C(N) \sqrt{|\Omega|} A^{N / 2}\|v\|_{L^{2}(\bar{\Omega})} \geq \frac{\|v\|_{C^{0}\left(\overline{\mathbf{\Omega}}^{\prime}\right)}}{\left(1+B /\left(A\|v\|_{C^{0}\left(\bar{\Omega}^{\prime}\right)}\right)\right)^{N / 2}},
$$

for any connected component $\Omega^{\prime}$. Hence

$$
2^{-N / 2} C(N) \sqrt{|\Omega|} A^{N / 2}\|v\|_{L^{2}(\bar{\Omega})} \geq \frac{\|v\|_{C^{0}(\bar{\Omega})}}{\left(1+B /\left(A\|v\|_{\left.C^{0}(\bar{\Omega})\right)^{N / 2}}\right.\right.} .
$$

If $\|v\|_{C^{0}(\bar{\Omega})} \geq B / A$, then it follows from (2.11) that

$$
2^{-N / 2} C(N) \sqrt{|\Omega|} A^{N / 2}\|v\|_{L^{2}(\bar{\Omega})} \geq \frac{\|v\|_{C^{0}(\bar{\Omega})}}{2^{N / 2}},
$$

which implies (2.1).

Now let us prove Proposition 2.1 for the case of arbitrary $v \in C_{0}^{2}(\overline{\mathbf{\Omega}})$. For any $\varepsilon>0$ we can choose a function $v_{\varepsilon} \in C_{0}^{\infty}(\bar{\Omega})$ such that $\left\|v-v_{\varepsilon}\right\|_{C^{2}(\bar{\Omega})} \leq \varepsilon$. Hence

$$
\left|\Delta v_{\varepsilon}(x)\right| \leq A\left|v_{\varepsilon}(x)\right|+(B+(A+N) \varepsilon) .
$$

We already proved Proposition 2.1 for all $C^{\infty}$-smooth functions. So

$$
\left\|v_{\varepsilon}\right\|_{C^{0}(\bar{\Omega})} \leq \max \left\{\frac{B+(A+N) \varepsilon}{A}, C(N)|\Omega|^{1 / 2} A^{N / 2}\left\|v_{\varepsilon}\right\|_{L^{2}(\bar{\Omega})}\right\} .
$$

Passing to the limit at $\varepsilon \rightarrow 0$ we get (2.1). 
Proposition 2.2. A solution $u$ of the problem (1.3a-b) satisfying the inequality $\|u\|_{C^{0}(\bar{\Omega})} \leq S$ also satisfies the inequality

$$
|\operatorname{grad} u(x)| \leq w_{N}^{-1 / N}|\Omega|^{1 / N}\left(M_{0}(S)+\|h\|_{C^{0}(\bar{\Omega})}\right)
$$

for any $x \in \bar{\Omega}$.

Proof. Integrating (1.1) we get

$$
u(y)=\int_{\Omega} G(x, y)(f(u(x), x)-h(x)) d x,
$$

where $G(x, y)$ is the Green's function for $\Omega$. In the particular case $p=1$, Weinberger's estimate (cf. [2, Theorem 2.5]) implies that for all $x \in \bar{\Omega}$,

$$
\int_{\Omega}|\operatorname{grad} G(x, y)| d x \leq w_{N}^{-1 / N}|\Omega|^{1 / N} .
$$

But if $\|u\|_{C^{0}(\bar{\Omega})} \leq S$ then, by the definition of $M_{0}(S),|f(u(x), x)| \leq M_{0}(S)$. Hence

$$
\begin{aligned}
|\operatorname{grad} u(x)| & \leq\left(\int_{\Omega}|\operatorname{grad} G(x, y)| d y\right)\left(\max _{y \in \bar{\Omega}}|f(u(y), y)|+\|h\|_{C^{0}(\bar{\Omega})}\right) \\
& \leq w_{N}^{1 / N}|\Omega|^{1 / N}\left(M_{0}(S)+\|h\|_{C^{0}(\bar{\Omega})}\right) .
\end{aligned}
$$

Proposition 2.3. Let $u(x)$ be a linear combination of eigenfunctions of the operator $-\Delta+\varphi(x)$ in $L_{0}^{2}(\Omega)$ corresponding to eigenvalues $\lambda_{i}$ such that $\lambda_{i} \leq \Lambda$. (Here $\varphi(x) \in C^{2+\alpha}(\bar{\Omega}), \Lambda$ is a positive constant.) Denote the number of such eigenvalues counted with their multiplicities by $m(\Lambda)$. Then

(a)

$$
|u(x)| \leq C(N)\left[\|\varphi\|_{C^{0}(\bar{\Omega})}+\Lambda\right]^{N / 2}|\Omega|^{1 / 2} m(\Lambda)^{1 / 2}\|u\|_{L^{2}(\bar{\Omega})},
$$

$$
|\operatorname{grad} u(x)| \leq C(N) w_{N}^{-1 / N}|\Omega|^{1 / 2+1 / N} m^{1 / 2}(\Lambda)\left[\|\varphi\|_{C^{0}(\bar{\Omega})}+\Lambda\right]^{N / 2+1}\|u\|_{L^{2}(\bar{\Omega})},
$$

where $C(N)$ is given by the formula (2.2).

Proof. Let $u=\sum_{\left\{i \mid \lambda_{i} \leq \Lambda\right\}} \alpha_{i} u_{i}$, where $u_{i}$ are the eigenfunctions of the unit $L^{2}$-norm corresponding to the eigenvalues $\lambda_{i}$. For any of the functions $u_{i}$ the following estimates take place:

$$
\left|u_{i}(x)\right| \leq C(N) \sqrt{|\Omega|}\left(\Lambda+\|\varphi\|_{C^{0}(\bar{\Omega})}\right)^{N / 2},
$$

by Proposition 2.1. and

$$
\left|\operatorname{grad} u_{i}(x)\right| \leq w_{N}^{-1 / N}|\Omega|^{1 / N}\left(\Lambda+\|\varphi\|_{C^{0}(\bar{\Omega})}\right)\left\|u_{i}\right\|_{C^{0}(\bar{\Omega})},
$$

by Proposition 2.2. 
Now note that

$$
\begin{aligned}
\left|\sum_{i=1}^{m(\Lambda)} \alpha_{i} u_{i}(x)\right| & \leq\left(\sum_{1}^{m(\Lambda)}\left|\alpha_{i}\right|\right) \max _{i}\left|u_{i}(x)\right| \\
& \leq m^{1 / 2}(\Lambda) \max _{i}\left|u_{i}(x)\right| \times \sqrt{\sum_{1}^{m(\Lambda)}\left|\alpha_{i}\right|^{2}} \\
& =m^{1 / 2}(\Lambda) \max _{i}\left|u_{i}(x)\right|\|u\|_{L^{2}(\bar{\Omega})}
\end{aligned}
$$

Part (a) follow now from (2.15).

Similarly, part (b) can be deduced from (2.15), (2.16), and the inequality $\sum_{i=1}^{m(\Lambda)}\left|\alpha_{i}\right| \leq \sqrt{m(\Lambda)} \sqrt{\sum_{i=1}^{m(\Lambda)} \alpha_{i}^{2}}$.

The next proposition shows how many balls of a prescribed radius in $C^{0}(\bar{\Omega})$ are a priori sufficient to cover the set $Q_{S}$ defined as

$$
Q_{S}=\bigcup_{\left\{h \in C^{\alpha}(\bar{\Omega}) \mid\|h\|_{C^{0}(\bar{\Omega})} \leq \varepsilon\right\}}\left\{u \in C_{0}^{2+\alpha}(\bar{\Omega}) \mid\|u\|_{C^{0}(\bar{\Omega})} \leq S\right.
$$

and $u$ is a solution of $(1.3)\}$,

and considered as a subset of $C^{0}(\bar{\Omega})$. We assume here that $0<\varepsilon \leq 1$.

Proposition 2.4. There exists the constant $\chi(N$, diam $\Omega)$ depending only on $N$ but not on $\varepsilon, f, S, \rho(S)$ with the following property. Let $\rho(S)>0$ be given. Then there exists a cover of $Q_{S}$ by balls of the radius $\rho(S)$ in $C^{0}(\bar{\Omega})$ such that

(a) Denote the number of the balls of the cover by $N B(S)$. Then

$$
\ln N B(S) \leq \chi(N, \operatorname{diam} \Omega)\left(\frac{S\left(M_{0}(S)+1\right)}{\rho(S)}\right)^{N}(\operatorname{dil} \Omega)^{N} .
$$

(b) The centers of all the balls of the cover lie in $Q$.

Proof. It follows from Proposition 2.2 that for any $S$,

$$
\begin{aligned}
Q_{S} \subset Q_{S}^{\prime} \stackrel{d f}{=}\left\{u \in C^{1}(\bar{\Omega}) \mid\|u\|_{C^{0}(\bar{\Omega})} \leq S\right. \\
\left.\|D u\|_{C^{0}(\bar{\Omega})} \leq w_{N}^{-1 / N}|\Omega|^{1 / N} \times\left(M_{0}(S)+1\right)\right\} .
\end{aligned}
$$

The techniques from the Kolmogorov and Tihomirov work [9] used to prove Theorem XIV there can be used to construct the cover of the set $Q_{S}^{\prime}$ by balls of the radius $\frac{1}{2} \rho(S)$ with centers in $C^{0}(\bar{\Omega})$ satisfying part (a) of Proposition 2.4 for some constant $\chi(N, \operatorname{diam} \Omega)$. Let us sketch the idea of the construction.

Denote

$$
\omega_{N}^{-1 / N}|\Omega|^{1 / N}\left(M_{0}(S)+1\right)
$$

by $\widetilde{M}_{1}(S)$. Consider a $\rho(S) /\left(8\right.$ const $\left._{1}(N) \operatorname{dil} \Omega \widetilde{M}_{1}(S)\right)$-net $N T$ for $\bar{\Omega}$ and in $\bar{\Omega}$ such that all distances between points of the net are not less than

$$
\rho(S) /\left(8 \operatorname{const}_{1}(N) \operatorname{dil} \Omega \widetilde{M}_{1}(S)\right)
$$

(where $\operatorname{const}_{1}(N) \geq 1$ is a constant which will be defined later). It can be 
easily shown that there exists a net satisfying these conditions and containing const $_{2}\left(S\left(M_{1}(S)+1\right) \text { dil } \Omega / \rho(S)\right)^{N}$ points (where const $_{2}$ is some fixed constant depending only on $N$ and $\operatorname{diam} \Omega$ ). We assume that $N T$ is such a net.

For any function $u \in \Omega_{S}^{\prime}$ all its values on $\bar{\Omega}$ are defined up to the accuracy $\rho(S) / 8$ (actually, even up to the accuracy $\rho(S) /\left(8\right.$ const $\left._{1}(N)\right)$ ) by values of $u$ at points of the net. If only approximate values of $u$ at points of the net up to the accuracy $\rho(S) / 8$ are known, then the value of $u$ in a point of $\bar{\Omega}$ is defined up to the accuracy $\rho(S) / 4$. So, we consider only a discrete set $D=\left\{j \rho(S) / 8 \text { const }_{1}(N)\right\}_{j=0}^{\left[8 \text { const }_{1}(N) S / \rho(S)\right]}$ of possible values of $u$ in every point of the net. We will call a mapping $\psi$ from $N T$ to $D$ admissible if it can be obtained by the following way. Let $u$ be an arbitrary function from $Q_{S}^{\prime}$. For every point $x_{0} \in N T$ we define $\psi\left(x_{0}\right)$ as the closest to $u\left(x_{0}\right)$ point in $D$. We will call two points of the net close if the balls of radius $\rho(S) /\left(8\right.$ const $\left._{1}(N) \operatorname{dil} \Omega \widetilde{M}_{1}(S)\right)$ centered at these points have a nonempty intersection. Note that the difference of the values of any $u \in Q_{S}^{\prime}$ at any two close points does not exceed $\rho(S) /\left(4\right.$ const $\left._{1}(N)\right)$. So, a mapping $N T \rightarrow D$ is admissible only if its values in close points differ by not more than $\rho(S) /\left(2\right.$ const $\left._{1}(N)\right)$. Thus, the number of admissible mappings of $N T$ into $D$ does not exceed $\left(\left[8\right.\right.$ const $\left.\left._{1}(N) S / \rho(S)\right]+1\right) 9^{\text {const }_{2}\left(S\left(M_{0}(S)+1\right) \operatorname{dil} \Omega / \rho(S)\right)^{N}}$. Given a mapping $\psi: N T \rightarrow D$ such that its values in close points differ by not more than $\rho(S) /\left(2\right.$ const $\left._{1}(N)\right)$, we can construct, using a partition of unity, a function $A$ on all $\mathbb{R}^{N}$ which assumes prescribed values at prescribed points and has a gradient with the norm bounded by $\operatorname{const}_{3}(N) \operatorname{dil} \Omega \widetilde{M}_{1}(S)$. Here $\operatorname{const}_{3}(N)$ is a constant which does not depend on $\operatorname{const}_{1}(N)$. Now let const $_{1}(N)=\max \left\{\right.$ const $\left._{3}(N), 1\right\}$. It is easy to see that restrictions of all such constructed functions $A$ on $\bar{\Omega}$ form the set of the centers of the balls of a necessary cover of $Q_{S}^{\prime}$.

This cover will also be a cover of $Q_{S}$. Now consider only those balls of the cover which have nonempty intersections with $Q_{S}$. For every such ball $B$ we consider a point $x_{B} \in B \cap Q_{S}$ and a ball $B^{\prime}$ of the radius $\rho(S)$ and centered at $x_{B}$. The union of all such balls $B^{\prime}$ is a necessary cover.

\section{Generalized Lyapunov Schmidt PROCEdure}

In this section we introduce the direct sum decompositions briefly mentioned before. These decompositions will be used to reduce the problem (1.3) locally to a finite-dimensional equation $T_{0}(x)=0$. We find some upper bounds on the norms of the first and the second differentials of $T_{h}$. We also study here how $T_{h}$ depends on the right-hand side of the equation (1.3a).

Let $u_{0}$ be a solution of the problem (1.3) with a right-hand side $\bar{h}$ such that $\|\bar{h}\|_{C^{0}(\bar{\Omega})} \leq \varepsilon$. We consider the decomposition $C^{2+\alpha}(\bar{\Omega})=u_{0}+P_{1} \oplus P_{2}$, (regarding $C^{2+\alpha}(\bar{\Omega}), P_{1}, P_{2}$ as subspaces of $L^{2}(\bar{\Omega})$ ), where $P_{1}$ is a finitedimensional space spanned by all eigenfunctions of the operator $F^{\prime}\left(u_{0}\right)=-\Delta+$ $f_{u}^{\prime}\left(u_{0}(x), x\right)$ on $L_{0}^{2}(\bar{\Omega})$ corresponding to eigenvalues $\lambda$ such that $\lambda \leq a+$ $b$, where $a$ and $b$ are positive constants which will be defined later. This decomposition has the following useful properties: 
Proposition 3.1. Let $\left\|u_{0}\right\|_{C^{0}(\bar{\Omega})} \leq S$.

(1) There exists a constant $C_{11}$ depending only on $N$ such that

$$
\operatorname{dim} P_{1} \leq C_{11}\left(a+b+M_{1}(S)\right)^{N / 2}(\operatorname{diam} \Omega)^{N} .
$$

(2) For any $u \in C^{2+\alpha}(\bar{\Omega})$ such that $\left\|u-u_{0}\right\|_{L^{2}(\bar{\Omega})} \leq b / M_{2}(S)$, and $\|u\|_{C^{0}(\bar{\Omega})}$ $\leq S$, and for any $w \in P_{2}$ such that $\left.w\right|_{\partial \Omega}=0$;

$$
\left\|F^{\prime}(u) w\right\|_{L^{2}(\bar{\Omega})} \geq a\|w\|_{L^{2}(\bar{\Omega})} .
$$

(3) For any $u \in C^{2+\alpha}(\bar{\Omega})$ such that $\left\|u-u_{0}\right\|_{L^{2}(\bar{\Omega})} \leq b / M_{2}(S)$, and $\|u\|_{C^{0}(\bar{\Omega})}$ $\leq S$, and for any $w \in P_{2}$ such that $\left.w\right|_{\partial \Omega}=0$;

$$
\begin{aligned}
\left\|F^{\prime}(u) w\right\|_{C^{0}(\bar{\Omega})} \geq\|w\|_{C^{0}(\bar{\Omega})} \min \left\{M_{1}(S)+a+b,\right. \\
\left.\frac{a}{C(N)|\Omega|\left(M_{1}(S)+a+b\right)^{N / 2}}\right\} .
\end{aligned}
$$

(4) Every function $h \in P_{1}$ satisfies the inequality

(3.4) $\|h\|_{C^{1}(\bar{\Omega})} \leq \operatorname{const}(N, \operatorname{diam} \Omega) \cdot\left(\operatorname{dim} P_{1}\right)^{1 / 2}\|h\|_{L^{2}(\bar{\Omega})}\left(M_{1}(S)+a+b\right)^{N / 2+1}$, with an appropriate constant $\operatorname{const}(N, \operatorname{diam} \Omega)$.

Proof. To prove (3.1) we use the monotonicity properties of eigenvalues. First note that for every $i$,

$$
\lambda_{i}\left(-\Delta+f_{u}^{\prime}\left(u_{0}(x), x\right)\right) \geq \lambda_{i}\left(-\Delta-M_{1}(S)\right) .
$$

Furthermore, $\lambda_{i}\left(-\Delta-M_{1}(S)\right)$ can be minorized by the $i$ th eigenvalue of $-\Delta-M_{1}(S)$ on $L_{0}^{2}\left(\Omega^{\prime}\right)$, where $\Omega^{\prime}$ is a ball of radius diam $\Omega$ comprising $\Omega$. This yields (3.1).

To show (3.2) note that by the definition of $P_{2}$ for any $v \in P_{2}$ such that $\left.v\right|_{\partial \Omega}=0$,

$$
\left\|F^{\prime}\left(u_{0}\right) v\right\|_{L^{2}(\bar{\Omega})} \geq(a+b)\|v\|_{L^{2}(\bar{\Omega})} .
$$

Hence for any $u$ such that $\left\|u-u_{0}\right\|_{L^{2}(\bar{\Omega})} \leq b / M_{2}(S)$,

$$
\begin{gathered}
\left\|F^{\prime}(u) v\right\|_{L^{2}(\bar{\Omega})} \geq\left\|F^{\prime}\left(u_{0}\right) v\right\|_{L^{2}(\bar{\Omega})}-\left\|\left(F^{\prime}(u)-F^{\prime}\left(u_{0}\right)\right) v\right\|_{L^{2}(\bar{\Omega})} \\
\geq\left(b+a-M_{2}(S) \frac{b}{M_{2}(S)}\right)\|v\|_{L^{2}(\bar{\Omega})}=a\|v\|_{L^{2}(\bar{\Omega})} .
\end{gathered}
$$

Here we used the obvious fact that $\left\|D^{2} F(u)\right\| \leq \max _{x \in \bar{\Omega}}\left|f_{u u}^{\prime \prime}(u(x), x)\right|$. This completes the proof of (3.2).

In order to prove (3.3) denote $F^{\prime}(u) w=-\Delta w+f_{u}^{\prime}(u(x), x) w$ by $\delta(x)$. So,

$$
\begin{aligned}
|\Delta w(x)| & \leq M_{1}(S)|w(x)|+\|\delta\|_{C^{0}(\bar{\Omega})} \\
& <\left(M_{1}(S)+a+b\right)|w(x)|+\|\delta\|_{C^{0}(\bar{\Omega})} .
\end{aligned}
$$

It follows from Proposition 2.1 that

$$
\|w\|_{C^{0}(\bar{\Omega})} \leq \max \left\{\frac{\|\delta(x)\|_{C^{0}(\bar{\Omega})}}{M_{1}(S)+a+b}, C(N) \sqrt{|\Omega|}\left(M_{1}(S)+a+b\right)^{N / 2}\|w\|_{L^{2}(\bar{\Omega})}\right\} .
$$


But according to (3.2)

$$
\|w\|_{L^{2}(\bar{\Omega})} \leq\|\delta\|_{L^{2}(\bar{\Omega})} / a \leq\|\delta\|_{C^{0}(\bar{\Omega})} \frac{\sqrt{|\Omega|}}{a} .
$$

Substituting this inequality into (3.8) and rewriting the resulting inequality as the estimate on $\|\delta\|_{C^{0}(\bar{\Omega})}$ we get $(3.3)$.

The part (4) follows immediately from Proposition 2.3.

Below $a$ and $b$ in the definition of the direct sum decomposition will depend on the upper bound $S$ for $\left\|u_{0}\right\|_{C^{0}(\bar{\Omega})}$ and will be denoted $a(S)$ and $b(S)$.

We need a quantitative version of the Implicit Function Theorem in Banach spaces. Proposition 3.2 is a modification of the Implicit Function Theorem from [10, p. 59].

Let $X, Y$ and $Z$ be Banach spaces and $G$ a $C^{2+\alpha}$-smooth mapping $X \times Y \rightarrow$ $Z \quad(\alpha \geq 0)$. Let $x_{0} \in X, y_{0} \in Y, G\left(x_{0}, y_{0}\right)=z_{0}$.

Suppose $\left\|D G_{y}\left(x_{0}, y_{0}\right)\right\| \leq C_{1},\left\|D^{2} G(x, y)\right\| \leq C_{2}$ for $x \in B_{r_{0}}\left(x_{0}\right), y \in$ $B_{r_{0}}\left(y_{0}\right)$, where $r_{0}, C_{1}, C_{2}$ are given positive numbers and $B_{r_{0}}\left(x_{0}\right), B_{r_{0}}\left(y_{0}\right)$ denote balls of radii $r_{0}$ around $x_{0}$ and $y_{0}$ in corresponding spaces. Let $A=$ $D G_{x}\left(x_{0}, y_{0}\right)$ be an isomorphism of $X$ onto $Z$.

Put

$$
\begin{aligned}
& \delta=\frac{3}{32\left\|A^{-1}\right\|^{2} C_{2}\left(2\left\|A^{-1}\right\| C_{1}+1\right)}, \\
& r=\min \left\{\frac{1}{4\left\|A^{-1}\right\| C_{2}\left(2\left\|A^{-1}\right\| C_{1}+1\right)}, r_{0}\right\} .
\end{aligned}
$$

Let $z_{1} \in Z,\left\|z_{1}-z_{0}\right\| \leq \delta$.

\section{Proposition 3.2.}

(i) There exists a unique (continuous) mapping $v^{\left(z_{1}\right)}: B_{r}\left(y_{0}\right) \rightarrow X$ such that $G\left(v^{\left(z_{1}\right)}(y), y\right) \equiv z_{1}$ and for any $y \in B_{r}\left(y_{0}\right)$,

$$
v^{\left(z_{1}\right)}(y) \in B_{\left(2\left\|A^{-1}\right\| C_{1}+1\right) r}\left(x_{0}\right) \text {. }
$$

(ii) $v^{\left(z_{1}\right)}$ is $C^{2+\alpha}$-smooth and

$$
D v_{y}^{\left(z_{1}\right)}(y)=-\left[D G_{x}\left(v^{\left(z_{1}\right)}(y), y\right)\right]^{-1} \circ D G_{y}\left(v^{\left(z_{1}\right)}(y), y\right) .
$$

(iii) For any $y \in B_{r}\left(y_{0}\right)$,

$$
\left\|v^{\left(z_{0}\right)}(y)-x_{0}\right\| \leq\left\|y-y_{0}\right\|\left(2\left\|A^{-1}\right\| C_{1}+1\right) .
$$

Proof. It follows closely the proof of the Implicit Function Theorem in [10, pp. 59-62]. One should only choose specific values of the constants mentioned there.

Namely, we may assume $x_{0}=0, y_{0}=0, z_{0}=0$. Let

$$
0<\varepsilon \leq \frac{1}{4\left\|A^{-1}\right\| C_{2}} .
$$

The inequality for $\varepsilon$ and (3.10) imply that

$$
\left\|D G_{x}(0,0)-D G_{x}(x, y)\right\| \leq \frac{1}{2\left\|A^{-1}\right\|},
$$

for any $x \in B_{\varepsilon}(0), y \in B_{r}(0)$. 
Hence, as it is shown in [10] for any triple $x_{1}, x_{2} \in B_{\varepsilon}(0), y \in B_{r}(0)$,

$$
\begin{aligned}
& \left\|\left(x_{1}-A^{-1}\left(G\left(x_{1}, y\right)-z_{1}\right)\right)-\left(x_{2}-A^{-1}\left(G\left(x_{2}, y\right)-z_{1}\right)\right)\right\| \\
& \quad \leq \frac{1}{2}\left\|x_{1}-x_{2}\right\| .
\end{aligned}
$$

Let the mapping $g$ be defined by the formula

$$
g(x, y)=x-A^{-1}\left(G(x, y)-z_{1}\right) .
$$

For each $y \in B_{r}(0)$ this mapping can be regarded as a mapping of $B_{\varepsilon}(0) \subset$ $X$ into $X$. The inequality (3.14) shows that this mapping is a contraction. Suppose now, that in addition to (3.13)

$$
r \leq \frac{\varepsilon}{2\left\|A^{-1}\right\| C_{1}+1}, \quad\left\|z_{1}\right\| \leq \frac{3 \varepsilon}{8\left\|A^{-1}\right\|\left(2\left\|A^{-1}\right\| C_{1}+1\right)} .
$$

Then

$$
\|g(0, y)\| \leq\left(\left\|z_{1}\right\|+C_{1} r+\frac{C_{2} r^{2}}{2}\right)\left\|A^{-1}\right\| \leq \frac{\varepsilon}{2} .
$$

Hence

$$
\|g(x, y)\| \leq\|g(0, y)\|+\|g(x, y)-g(0, y)\| \leq \frac{\varepsilon}{2}+\frac{\varepsilon}{2}=\varepsilon .
$$

Thus, for each $y \in B_{r}(0) g(\cdot, y)$ maps $B_{\varepsilon}(0) \subset X$ into itself. Hence, by the Strict Contraction Mapping Principle it has a unique fixed point. We can take this point to be $v^{\left(z_{1}\right)}(y)$. Note that we can choose $\varepsilon=\left(2\left\|A^{-1}\right\| C_{1}+1\right) r$, where $r$ is defined by (3.10). The continuity of $v^{\left(z_{1}\right)}(y)$ and (ii) are proven in [10].

To prove (3.12) note that if $\|y\|=r_{1}<r$, then we can take $r_{1}\left(2\left\|A^{-1}\right\| C_{1}+1\right)$ for $\varepsilon$, and this $\varepsilon$ will satisfy (3.13). The proof above shows that $v^{\left(z_{0}\right)}(y) \in$ $B_{\varepsilon}(0)$ also for this $\varepsilon$, which proves (3.12).

Suppose now that some $a(S), b(S)>0$ are fixed, $\left\|u_{0}\right\|_{C^{0}(\bar{\Omega})} \leq S, P_{1}$ and $P_{2}$ are defined as at the beginning of this section. Let us introduce the space

$$
P_{2}^{0}=\left\{u \in P_{2}|u|_{\partial \Omega}=0\right\},
$$

and denote by $m_{u_{0}}(S)$ an upper bound $C_{12}\left(a+b+M_{1}(S)\right)^{N / 2}$ on $\operatorname{dim} P_{1}$ which follows immediately from (3.1). Here the constant $C_{12}$ depends on $N$ and $\operatorname{diam} \Omega$ only.

Let $Q_{1}=F^{\prime}\left(u_{0}\right)\left(P_{1}\right) \oplus \operatorname{Coker} F^{\prime}\left(u_{0}\right)$ and $Q_{2}=F^{\prime}\left(u_{0}\right)\left(P_{2}^{0}\right)$. Obviously, $Q_{1} \oplus$ $Q_{2}=C^{\alpha}(\bar{\Omega})$.

We shall consider $P_{1}, P_{2}^{0}, Q_{1}, Q_{2}$ as normed spaces with $C^{0}$-norm or with $L^{2}$-norm. Correspondingly, linear mappings between these spaces will be equipped with $C^{0}$ or $L^{2}$-norm.

Let $\pi_{Q_{1}}, \pi_{Q_{2}}$ denote operators of orthogonal projection onto $Q_{1}$ and $Q_{2}$, respectively. Applying $\pi_{Q_{1}}$ and $\pi_{Q_{2}}$ to the problem (1.3) we can replace it by the following equivalent system:

$$
\left\{\begin{array}{l}
\pi_{Q_{1}} F\left(u_{1}, u_{2}\right)=\pi_{Q_{1}} h, \\
\pi_{Q_{2}} F\left(u_{1}, u_{2}\right)=\pi_{Q_{2}} h .
\end{array}\right.
$$


Now we want to apply Proposition 3.2 to the mapping $\pi_{Q_{2}} \circ F: P_{2}^{0} \times P_{1} \rightarrow Q_{2}$, where all three spaces $P_{2}^{0}, P_{1}$, and $Q_{2}$ are assumed to be equipped with $C^{0}$ norms. Proposition 3.1 (3) implies that

$$
\begin{aligned}
\left\|A^{-1}\right\| & \equiv\left\|\left(\left.D\left(\pi_{Q_{2}} \circ F\right)\right|_{P_{2}^{0}}\right)^{-1}\left(u_{0}\right)\right\|_{C^{0}} \\
& \leq \max \left\{\frac{1}{M_{1}(S)+a(S)+b(S)}, \frac{C(N)|\Omega|\left(M_{1}(S)+a(S)+b(S)\right)^{N / 2}}{a(S)}\right\},
\end{aligned}
$$

where $C(N)$ is given by $(2.2)$.

$$
\begin{aligned}
&\left\|D G_{y}\left(x_{0}, y_{0}\right)\right\| \equiv \sup _{\left\{v \in P_{1} \mid\|v\|_{C^{0}(\bar{\Omega})}=1\right\}}\left\|-\Delta v+f_{u}^{\prime}\left(u_{0}(x), x\right) v\right\|_{C^{0}(\bar{\Omega})} \\
& \leq \sup _{\left\{v \in P_{1} \mid\|v\|_{L^{2}(\bar{\Omega})} \leq \sqrt{|\Omega|}\right\}}\left\|-\Delta v+f_{u}^{\prime}\left(u_{0}(x), x\right) v\right\|_{L^{2}(\bar{\Omega})} \\
& \cdot C(N) \sqrt{|\Omega|} m_{u_{0}}(S)^{1 / 2}\left(M_{1}(S)+a(S)+b(S)\right)^{N / 2},
\end{aligned}
$$

by Proposition 2.3(a). Hence

$$
\begin{aligned}
\left\|D G_{y}\left(x_{0}, y_{0}\right)\right\| & \leq C(N)|\Omega| m_{u_{0}}(S)^{1 / 2}\left(M_{1}(S)+a(S)+b(S)\right)^{N / 2+1} \\
& \leq \widetilde{C}(N, \operatorname{diam} \Omega)\left(M_{1}(S)+a(S)+b(S)\right)^{3 N / 4+1}
\end{aligned}
$$

for an appropriate constant $\widetilde{C}(N, \operatorname{diam} \Omega)$.

The right-hand side of (3.19) can be taken for $C_{1}$. And

$$
\left\|D^{2} G(x, y)\right\| \leq M_{2}(S) \leq \bar{M}_{2}(S) .
$$

We take $S-\left\|u_{0}\right\|_{C^{0}(\bar{\Omega})}$ for $r_{0}$. Thus, formulae (3.9), (3.10) can be written as follows. Denote

$$
k_{a b}(S)=\max \left\{\frac{1}{M_{1}(S)+a(S)+b(S)}, \frac{C(N)|\Omega|\left(M_{1}(S)+a(S)+b(S)\right)^{N / 2}}{a(S)}\right\} .
$$

Then for some positive $C_{3}(N,|\Omega|), C_{4}(N,|\Omega|)$ which can be written explicitly (3.22)

$$
\delta(S)=C_{3}(N,|\Omega|) \frac{1}{k_{a b}^{2}(S) \bar{M}_{2}(S)\left(k_{a b}(S)\left(M_{1}(S)+a(S)+b(S)\right)^{3 N / 4+1}+1\right)},
$$

and

$$
r(S)=C_{4}(N,|\Omega|) \frac{1}{k_{a b}(S) \bar{M}_{2}(S)\left(k_{a b}(S)\left(M_{1}(S)+a(S)+b(S)\right)^{3 N / 4+1}+1\right)},
$$

can be considered as $\delta$ and $r$ in the statement of Proposition 3.2 if only $r(S) \leq S-\left\|u_{0}\right\|_{C^{0}(\bar{\Omega})}$. The application of Proposition 3.2 yields the existence of mappings: $\psi_{\tilde{h}}: B_{r(S)}^{P_{1}}\left(\pi_{P_{1}}\left(u_{0}\right)\right) \rightarrow P_{2}^{0}$, where $B_{r(S)}^{P_{1}}\left(\pi_{P_{1}}\left(u_{0}\right)\right)$ denotes the $C^{0}$ norm ball around $\pi_{P_{1}}\left(u_{0}\right)$ in $P_{1}$ of radius $r(S)$ and $\|\tilde{h}-\bar{h}\|_{C^{0}(\bar{\Omega})} \leq \delta(S)$, such that

$$
\pi_{Q_{2}} F\left(u_{1}, \psi_{\tilde{h}}\left(u_{1}\right)\right)=\pi_{Q_{2}} \tilde{h}
$$


Moreover, $\psi_{\hat{h}}\left(u_{1}\right)$ is the unique solution $u_{2}$ of (3.24) in the ball $B_{\left(2\left\|A^{-1}\right\| C_{1}+1\right) r}$ around $\pi_{P_{2}^{0}}\left(u_{0}\right)$ in $P_{2}^{0}$ (see (3.18), (3.19) for the estimates of $\left.\left\|A^{-1}\right\|, C_{1}\right)$. Let $T_{\tilde{h}}: B_{r(S)}^{P_{1}}\left(\pi_{P_{1}}\left(u_{0}\right)\right) \rightarrow Q_{1}$ be defined by the formula

$$
T_{\tilde{h}}\left(u_{1}\right)=\pi_{Q_{1}} F\left(u_{1}, \psi_{\tilde{h}}\left(u_{1}\right)\right)-\pi_{Q_{1}} \tilde{h} .
$$

We have proven the following proposition:

Proposition 3.3. Let $F\left(u_{0}\right)=\bar{h}$. Suppose $\left\|u_{0}\right\|_{C^{0}(\bar{\Omega})} \leq S-r(S)$. For any $\tilde{h} \in C^{\alpha}(\bar{\Omega})$ such that $\|\bar{h}-\tilde{h}\|_{C^{0}(\bar{\Omega})} \leq \delta(S)$ the cardinality of the set of solutions $u \in C_{0}^{2+\alpha}(\bar{\Omega})$ of the boundary value problem $F(u)=\tilde{h}$ such that $\left\|u-u_{0}\right\|_{C^{0}(\bar{\Omega})} \leq$ $r(S)$ does not exceed the cardinality of the solution set of the equation $T_{\hat{h}}\left(u_{1}\right)=0$ in the finite-dimensional ball $B_{r(S)}^{P_{1}}\left(\pi_{P_{1}}\left(u_{0}\right)\right)$ in $P_{1}$.

Now we would like to estimate the first and second derivatives of $T_{\tilde{h}}$ and to obtain some information about the dependence of $T_{\tilde{h}}$ on $\tilde{h}$.

Let

$$
\Delta_{a b}(S)=2\left(k_{a b}(S)\left(M_{1}(S)+a(S)+b(S)\right)^{3 N / 4+1}+1\right) .
$$

It follows from Proposition 3.2 that

$$
\left\|u_{1}-\pi_{P_{1}}\left(u_{0}\right)\right\|_{C^{0}(\bar{\Omega})}+\left\|\psi_{\hat{h}}\left(u_{1}\right)-\pi_{P_{2}^{0}}\left(u_{0}\right)\right\|_{C^{0}(\bar{\Omega})} \leq r(S) \Delta_{a b}(S) .
$$

Note also that $F^{\prime}\left(u_{0}\right)$ is a selfadjoint operator on $L_{0}^{2}(\bar{\Omega})$. Hence $C_{0}^{2+\alpha}(\Omega) \supset$ $\operatorname{Ker} F^{\prime}\left(u_{0}\right)=\operatorname{Coker} F^{\prime}\left(u_{0}\right)$. Thus, $P_{1} \equiv Q_{1}$, and all estimates on elements of $P_{1}$ hold automatically for elements of $Q_{1}$.

Proposition 3.4. Assume that

$$
r(S) \leq \frac{b(S)}{M_{2}(S) \Delta_{a b}(S)} .
$$

Then for any such $\tilde{h} \in C^{\alpha}(\bar{\Omega})$ that

$$
\|\tilde{h}-\bar{h}\|_{C^{0}(\bar{\Omega})} \leq \delta(S), \quad \text { for any } u_{1} \in B_{r(S)}^{P_{1}}\left(\pi_{P_{1}}\left(u_{0}\right)\right):
$$

(a)

$$
\left\|D T_{\hat{h}}\left(u_{1}\right)\right\|_{L^{2}(\bar{\Omega})} \leq \max \left\{M_{1}(S), a(S)+b(S)\right\}+\frac{b(S)}{a(S)}(a(S)+b(S)) .
$$

(b)

$$
\left\|D^{2} T_{\hat{h}}\left(u_{1}\right)\right\|_{L^{2}(\bar{\Omega})} \leq M_{2}(S)\left(1+\frac{b(S)}{a(S)}\right)^{3} .
$$

Suppose now that $\delta_{1}>0$ is a given constant and $h_{1}, h_{2} \in C^{\alpha}(\bar{\Omega})$ satisfy $\left\|h_{i}-\bar{h}\right\|_{C^{0}(\bar{\Omega})} \leq \delta(S) \quad(i \in\{1,2\}),\left\|h_{1}-h_{2}\right\|_{C^{0}(\bar{\Omega})} \leq \delta_{1}$. Then

$$
\left\|T_{h_{1}}\left(u_{1}\right)-T_{h_{2}}\left(u_{1}\right)\right\|_{L^{2}(\bar{\Omega})} \leq \delta_{1}\left(1+\frac{b(S)}{a(S)}\right) \sqrt{|\bar{\Omega}|},
$$


(d)

$$
\left\|D T_{h_{1}}\left(u_{1}\right)-D T_{h_{2}}\left(u_{1}\right)\right\|_{L^{2}(\bar{\Omega})} \leq \delta_{1} \frac{M_{2}(S)}{a(S)}\left(1+\frac{b(S)}{a(S)}\right)^{2} \sqrt{|\Omega|} .
$$

Proof. Note that by the Implicit Function Theorem (Proposition 3.2(ii))

$$
D \psi_{\tilde{h}}\left(u_{1}\right)=-\left(\pi_{Q_{2}} D F_{P_{2}^{0}}\left(u_{1}, u_{2}\right)\right)^{-1}\left(\pi_{Q_{2}} D F_{P_{1}}\left(u_{1}, u_{2}\right)\right),
$$

where $u_{2}$ denotes $\psi_{\hat{h}}\left(u_{1}\right)$.

Hence

$$
\begin{aligned}
D T_{\tilde{h}}\left(u_{1}\right)= & \left(\pi_{Q_{1}} D F_{P_{1}}\left(u_{1}, u_{2}\right)\right)-\left(\pi_{Q_{1}} D F_{P_{2}^{0}}\left(u_{1}, u_{2}\right)\right) \\
& \times\left(\pi_{Q_{2}} D F_{P_{2}^{0}}\left(u_{1}, u_{2}\right)\right)^{-1}\left(\pi_{Q_{2}} D F_{P_{1}}\left(u_{1}, u_{2}\right)\right) .
\end{aligned}
$$

Estimate now $\left\|\pi_{Q_{i}} D F_{P_{1}}\left(u_{1}, u_{2}\right)\right\|_{L^{2}}, i \in\{1,2\}$, and $\left\|\pi_{Q_{1}} D F_{P_{2}^{0}}\left(u_{1}, u_{2}\right)\right\|_{L^{2}}$ for $u_{1} \in B_{r(S)}^{P_{1}}\left(\pi_{P_{1}}\left(u_{0}\right)\right)$. For any $v \in P_{1}$,

$$
\begin{gathered}
\left\|\pi_{Q_{2}} D F_{P_{1}}\left(u_{1}, u_{2}\right) v\right\|_{L^{2}(\bar{\Omega})} \leq\left\|\left(\pi_{Q_{2}} D F_{P_{1}}\left(u_{1}, u_{2}\right)-\pi_{Q_{2}} D F_{P_{1}}\left(u_{0}\right)\right) v\right\|_{L^{2}(\bar{\Omega})} \\
=\left\|\pi_{Q_{2}}\left(\left(f_{u}^{\prime}\left(u_{1}(x)+u_{2}(x), x\right)-f_{u}^{\prime}\left(u_{0}(x), x\right)\right) v\right)\right\|_{L^{2}(\bar{\Omega})} .
\end{gathered}
$$

Hence

$$
\begin{aligned}
\left\|\pi_{Q_{2}} D F_{P_{1}}\left(u_{1}, u_{2}\right)\right\|_{L^{2}} & \leq M_{2}(S)\left\|u_{1}-\pi_{P_{1}}\left(u_{0}\right)+u_{2}-\pi_{P_{2}^{0}}\left(u_{0}\right)\right\|_{C^{0}(\bar{\Omega})} \\
& \leq M_{2}(S) r(S) \Delta_{a b}(S) .
\end{aligned}
$$

Here we used (3.29) (recall that $\left.u_{2}=\psi_{\hat{h}}\left(u_{1}\right)\right)$. Thus,

$$
\left\|\pi_{Q_{2}} D F_{P_{1}}\left(u_{1}, u_{2}\right)\right\|_{L^{2}} \leq M_{2}(S) r(S) \Delta_{a b}(S) .
$$

Similarly,

$$
\begin{gathered}
\left\|\pi_{Q_{1}} D F_{P_{1}}\left(u_{1}, u_{2}\right)\right\|_{L^{2}} \leq\left\|\pi_{Q_{1}} D F_{P_{1}}\left(\pi_{P_{1}}\left(u_{0}\right)+\pi_{P_{2}^{0}}\left(u_{0}\right)\right)\right\|_{L^{2}} \\
+M_{2}(S)\left\|u_{1}-\pi_{P_{1}}\left(u_{0}\right)+u_{2}-\pi_{P_{2}^{0}}\left(u_{0}\right)\right\|_{C^{0}(\bar{\Omega})} \\
\leq \max \left\{M_{1}(S), a(S)+b(S)\right\}+M_{2}(S) r(S) \Delta_{a b}(S) . \\
\left\|\pi_{Q_{1}} D F_{P_{2}^{0}}\left(u_{1}, u_{2}\right)\right\|_{L^{2}} \leq M_{2}(S) r(S) \Delta_{a b}(S) .
\end{gathered}
$$

Note that because of the assumption (3.30)

$$
M_{2}(S) r(S) \Delta_{a b}(S) \leq b(S) .
$$

Hence

(3.40) $\left\|\left(\pi_{Q_{2}} D F_{P_{2}^{0}}\left(u_{1}, u_{2}\right)\right)^{-1}\right\|_{L^{2}} \leq \frac{1}{a(S)+b(S)-M_{2}(S) r(S) \Delta_{a b}(S)} \leq \frac{1}{a(S)}$.

Formulae (3.35)-(3.40) imply that

$$
\left\|D T_{\hat{h}}\left(u_{1}\right)\right\|_{L^{2}} \leq \max \left\{M_{1}(S), a(S)+b(S)\right\}+b(S)+\frac{b^{2}(S)}{a(S)} .
$$

To prove $(b)$ note that

$$
\begin{aligned}
\left\|D^{2} \psi_{\hat{h}}\left(u_{1}\right)\right\|_{L^{2}} \leq & \left\|\left(\pi_{Q_{2}} D F_{P_{2}^{0}}\left(u_{1}, u_{2}\right)\right)^{-1}\right\|_{L^{2}} \\
& \times\left(\left\|\pi_{Q_{2}} D^{2} F_{P_{1} P_{1}}\left(u_{1}, u_{2}\right)\right\|_{L^{2}}+2\left\|\pi_{Q_{2}} D^{2} F_{P_{1} P_{2}^{0}}\left(u_{1}, u_{2}\right)\right\|_{L^{2}}\right. \\
& \left.\times\left\|D \psi_{\grave{h}}\left(u_{1}\right)\right\|_{L^{2}}+\left\|\pi_{Q_{2}} D^{2} F_{P_{2}^{0} P_{2}^{0}}\right\|_{L^{2}}\left\|D \psi_{\hat{h}}\left(u_{1}\right)\right\|_{L^{2}}^{2}\right) .
\end{aligned}
$$


Hence

$$
\left\|D^{2} \psi_{\tilde{h}}\left(u_{1}\right)\right\|_{L^{2}} \leq \frac{M_{2}(S)}{a(S)}\left(1+\frac{b(S)}{a(S)}\right)^{2} .
$$

Now, differentiating (3.25) and taking into account (3.36-3.40), (3.42) we get

$$
\begin{aligned}
\left\|D^{2} T_{\hat{h}}\right\|_{L^{2}} & \leq \frac{M_{2}(S)}{a(S)}\left(1+\frac{b(S)}{a(S)}\right)^{2} \cdot b(S)+M_{2}(S)\left(1+\frac{b(S)}{a(S)}\right)^{2} \\
& =M_{2}(S)\left(1+\frac{b(S)}{a(S)}\right)^{3},
\end{aligned}
$$

which proves (b).

Let now $h_{1}, h_{2}$ satisfy the constraints of Proposition 3.4. Then

$$
\pi_{Q_{2}} F\left(u_{1}, \psi_{h_{1}}\left(u_{1}\right)\right)-\pi_{Q_{2}} F\left(u_{1}, \psi_{h_{2}}\left(u_{1}\right)\right)=\pi_{Q_{2}}\left(h_{1}-h_{2}\right) .
$$

So,

$$
\begin{aligned}
& \pi_{Q_{2}}\left(h_{1}-h_{2}\right) \\
& \quad=\int_{0}^{1} \pi_{Q_{2}} D_{P_{2}^{0}} F\left(u_{1}, t \psi_{h_{1}}\left(u_{1}\right)+(1-t) \psi_{h_{2}}\left(u_{1}\right)\right)\left(\psi_{h_{1}}\left(u_{1}\right)-\psi_{h_{2}}\left(u_{2}\right)\right) d t .
\end{aligned}
$$

Note that

$$
\pi_{Q_{2}} D_{P_{2}^{0}} F\left(\pi_{P_{1}}\left(u_{0}\right), \psi_{\bar{h}}\left(\pi_{P_{1}}\left(u_{0}\right)\right)\right)=D_{P_{2}^{0}} F\left(\pi_{P_{1}}\left(u_{0}\right), \psi_{\bar{h}}\left(\pi_{P_{1}}\left(u_{0}\right)\right)\right),
$$

(here $\psi_{\bar{h}}$ means $\psi_{\tilde{h}}$ for $\tilde{h} \equiv \bar{h}$ ) and for any $v \in P_{2}^{0}$,

$$
\left\|D_{P_{2}^{0}} F\left(\pi_{P_{1}}\left(u_{0}\right), \psi_{\bar{h}}\left(\pi_{P_{1}}\left(u_{0}\right)\right)\right) v\right\|_{L^{2}(\bar{\Omega})} \geq(a(S)+b(S))\|v\|_{L^{2}(\bar{\Omega})} .
$$

Thus

$$
\begin{aligned}
\sqrt{|\Omega|} & \delta_{1}\left\|\psi_{h_{1}}\left(u_{1}\right)-\psi_{h_{2}}\left(u_{1}\right)\right\|_{L^{2}(\bar{\Omega})} \\
\geq & \left(\pi_{Q_{2}}\left(h_{1}-h_{2}\right), \psi_{h_{1}}\left(u_{1}\right)-\psi_{h_{2}}\left(u_{1}\right)\right)_{L^{2}(\bar{\Omega})} \\
= & \left(D_{P_{2}^{0}} F\left(\pi_{P_{1}}\left(u_{0}\right), \psi_{\bar{h}}\left(\pi_{P_{1}}\left(u_{0}\right)\right)\right)\left(\psi_{h_{1}}\left(u_{1}\right)-\psi_{h_{2}}\left(u_{1}\right)\right), \psi_{h_{1}}\left(u_{1}\right)-\psi_{h_{2}}\left(u_{1}\right)\right)_{L^{2}(\bar{\Omega})} \\
& +\left(\int _ { 0 } ^ { 1 } \pi _ { Q _ { 2 } } \left(\left[f^{\prime}\left(u_{1}+t \psi_{h_{1}}\left(u_{1}\right)+(1-t) \psi_{h_{2}}\left(u_{1}\right), x\right)-f^{\prime}\left(u_{0}, x\right)\right]\right.\right. \\
& \left.\left.\times\left(\psi_{h_{1}}\left(u_{1}\right)-\psi_{h_{2}}\left(u_{1}\right)\right)\right) d t, \psi_{h_{1}}\left(u_{1}\right)-\psi_{h_{2}}\left(u_{1}\right)\right)_{L^{2}(\bar{\Omega})} \\
\geq & (a(S)+b(S))\left\|\psi_{h_{1}}\left(u_{1}\right)-\psi_{h_{2}}\left(u_{1}\right)\right\|_{L^{2}(\bar{\Omega})}^{2}-b(S)\left\|\psi_{h_{1}}\left(u_{1}\right)-\psi_{h_{2}}\left(u_{1}\right)\right\|_{L^{2}(\bar{\Omega})}^{2} \\
= & a(S)\left\|\psi_{h_{1}}\left(u_{1}\right)-\psi_{h_{2}}\left(u_{1}\right)\right\|_{L^{2}(\bar{\Omega})}^{2}
\end{aligned}
$$

Therefore,

$$
\left\|\psi_{h_{1}}\left(u_{1}\right)-\psi_{h_{2}}\left(u_{1}\right)\right\|_{L^{2}(\bar{\Omega})} \leq \delta_{1}|\Omega|^{1 / 2} / a(S)
$$

Now

$$
\begin{aligned}
& \left\|T_{h_{1}}\left(u_{1}\right)-T_{h_{2}}\left(u_{1}\right)\right\|_{L^{2}(\bar{\Omega})} \\
& \quad=\| \pi_{Q_{1}}\left(F\left(u_{1}, \psi_{h_{1}}\left(u_{1}\right)\right)-F\left(u_{1}, \psi_{h_{2}}\left(u_{1}\right)\right)-\pi_{Q_{1}}\left(h_{1}-h_{2}\right) \|_{L^{2}(\bar{\Omega})}\right. \\
& \quad \leq b(S) \frac{\delta_{1} \sqrt{|\Omega|}}{a(S)}+\delta_{1} \sqrt{|\Omega|}=\delta_{1}|\Omega|^{1 / 2}\left(1+\frac{b(S)}{a(S)}\right) .
\end{aligned}
$$


(Here we used (3.38), (3.39), (3.44).) This proves part (c) of the proposition. It follows from (3.35), (3.44) and (3.36)-(3.40) that

$$
\left\|D T_{h_{1}}\left(u_{1}\right)-D T_{h_{2}}\left(u_{1}\right)\right\|_{L^{2}} \leq\left(\frac{\delta_{1}|\Omega|^{1 / 2}}{a(S)}\right) M_{2}(S)\left(1+2 \frac{b(S)}{a(S)}+\frac{b^{2}(S)}{a^{2}(S)}\right) .
$$

This implies (3.34).

Now we fix $a(S), b(S)$. Let

$$
a(S)=\bar{M}_{2}(S), \quad b(S)=C_{5}(N,|\Omega|) \bar{M}_{2}(S),
$$

where $\bar{M}_{2}(S)$ is defined by $(1.5)$ and the constant $C_{5}(N,|\Omega|)$ will be defined later. Then there exist constants $C_{6}(N,|\Omega|), \bar{C}_{6}(N,|\Omega|), C_{7}(N,|\Omega|)$, $C_{8}(N,|\Omega|), \bar{C}_{8}(N,|\Omega|)$ such that

$$
\begin{gathered}
C_{6}(N,|\Omega|)\left(\bar{M}_{2}(S)\right)^{N / 2-1} \leq k_{a b}(S) \leq \bar{C}_{6}(N,|\Omega|)\left(\bar{M}_{2}(S)\right)^{N / 2-1} \\
\delta(S) \geq C_{7}(N,|\Omega|)\left(\bar{M}_{2}(S)\right)^{-(9 N / 4-1)} \\
r(S) \geq C_{8}(N,|\Omega|)\left(\bar{M}_{2}(S)\right)^{-7 N / 4}
\end{gathered}
$$

and

$$
r(S) \leq \bar{C}_{8}(N,|\Omega|)\left(\bar{M}_{2}(S)\right)^{-7 N / 4},
$$

provided that $\left\|u_{0}\right\|_{C^{0}(\bar{\Omega})} \leq S-\bar{C}_{8}(N,|\Omega|)\left(\bar{M}_{2}(S)\right)^{-7 N / 4}$. Hence, asymptotically, when $S \rightarrow+\infty$,

$$
r(S) \leq \frac{b(S)}{M_{2}(S) \Delta_{a b}(S)},
$$

because the right-hand side $\geq C_{9}(N,|\Omega|)\left(\bar{M}_{2}(S)\right)^{-5 N / 4}$. Here we used

$$
\Delta_{a b}(S) \leq C_{10}(N,|\Omega|)\left(\bar{M}_{2}(S)\right)^{5 N / 4} .
$$

Formulae for $C_{6}(N,|\Omega|)-C_{10}(N,|\Omega|)$ can written explicitly but they include the constant $C_{5}(N,|\Omega|)$ which is not yet defined. It can be easily seen that for any fixed $S, r(S) M_{2}(S) \Delta_{a b}(S) / b(S) \rightarrow 0$ when $C_{5}(N,|\Omega|) \rightarrow+\infty$. Hence we can find and fix a large enough $C_{5}(N,|\Omega|)$ such that (3.30) holds for all $S$. Now we can conclude from Proposition 3.4 that there exist constants $C_{14}(N,|\Omega|), C_{15}(N,|\Omega|), C_{16}(N,|\Omega|), C_{17}(N,|\Omega|)$ such that

$$
\begin{aligned}
& \left\|D T_{\tilde{h}}\left(u_{1}\right)\right\|_{L^{2}} \leq C_{14}(N,|\Omega|) \bar{M}_{2}(S), \\
& \left\|D^{2} T_{\tilde{h}}\left(u_{1}\right)\right\|_{L^{2}} \leq C_{15}(N,|\Omega|) \bar{M}_{2}(S),
\end{aligned}
$$

and for $h_{1}, h_{2}$ such that $\left\|h_{1}-h_{2}\right\|_{C^{0}(\bar{\Omega})} \leq \delta_{1},\left\|h_{i}-\bar{h}\right\|_{C^{0}(\bar{\Omega})} \leq \delta(S) \quad(i \in\{1,2\})$

$$
\begin{aligned}
& \left\|T_{h_{1}}\left(u_{1}\right)-T_{h_{2}}\left(u_{1}\right)\right\|_{L^{2}(\bar{\Omega})} \leq C_{16}(N,|\Omega|) \delta_{1}, \\
& \left\|D T_{h_{1}}\left(u_{1}\right)-D T_{h_{2}}\left(u_{1}\right)\right\|_{L^{2}} \leq C_{17}(N,|\Omega|) \delta_{1} .
\end{aligned}
$$

\section{NUMBER OF SOLUTIONS OF A SET OF FINITE-DIMENSIONAL EQUATIONS WITH A COMMON GENERIC RIGHT-HAND SIDE} lem

$$
\left\{\begin{array}{l}
-\Delta u+f(u, x)=\tilde{h}(x) \text { in } \Omega, \\
\left.u\right|_{\partial \Omega}=0
\end{array}\right.
$$


(where $\left.\|\tilde{h}-h\|_{C^{0}(\bar{\Omega})} \leq \delta(S),\|h\|_{C^{0}(\bar{\Omega})} \leq \varepsilon\right)$ such that $\left\|u-u_{0}\right\|_{C^{0}(\bar{\Omega})} \leq r(S)$ does not exceed the number of solutions of the finite-dimensional equation

$$
\pi_{Q_{1}} F\left(u_{1}, \psi_{\tilde{h}}\left(u_{1}\right)\right)=\pi_{Q_{1}} \tilde{h},
$$

in the ball $B_{r(S)}^{P_{1}}\left(\pi_{P_{1}}\left(u_{0}\right)\right)$ in $P_{1}$. Recall that $u_{0}$ is a solution of $(1.3 \mathrm{a}, \mathbf{b})$ such that $\left\|u_{0}\right\|_{C^{0}(\bar{\Omega})} \leq S-r(S) ; \delta(S)$ and $r(S)$ are given by (3.22) and (3.23). When $a(S)$ and $b(S)$ are defined by the formula (3.46), $\delta(S)$ and $r(S)$ satisfy the inequalities (3.48) and (3.49), (3.50), respectively.

We would like to choose $\tilde{h}$ in such a way to make the number of solutions of the perturbed problem (4.1) small. This means that we should choose $\tilde{h}$ to make the numbers of solutions of a set of finite-dimensional equations (4.2) small. (Every equation in this set corresponds to a function $u_{0}$ which is an element of a certain net in the a priori solution set. See $\S 5$ for more details.) Thus we come to the problem a simplified version of which can be stated as follows.

Let $\mathbb{R}_{i}^{m} \subset \mathbb{R}^{p}, i \in\{1, \ldots, K\}$, be $K$ linear subspaces of a Euclidean space $\mathbb{R}^{p}$. Let $\varphi_{i}: \mathbb{R}^{m} \supset B_{\rho} \rightarrow \mathbb{R}_{i}^{m}$ be $K$ maps from a ball of a radius $\rho$ around the origin in $\mathbb{R}^{m}$ into $\mathbb{R}_{i}^{m}$. We would like to find $\xi \in \mathbb{R}^{p}$ such that $|\xi| \leq \varepsilon$ and the total number of solutions $\widetilde{N}_{\xi}$ of all equations $\varphi_{i}(x)=\pi_{\mathbb{R}_{i}^{m}} \xi$ (considered separately) is the smallest possible. Suppose such $\xi$ is found. What can be said about $\tilde{N}_{\xi}$ ?

The case $K=1$ of this problem was considered in [16] (even in much more greater generality). The considerations there were grounded on the quantitative version of the Sard's Theorem proven in [14] (see also [15]). We present here an immediate corollary from this theorem (Theorem 4.1). The results of [16] can be easily generalized for the case of $K>1$ and an upper bound on $\widetilde{N}_{\xi}$ in the mentioned problem can be found.

However, we should deal with a bit more intricate technical problem below. One difference is due to the fact that the mapping of the left-hand side of the equation (4.2) depends on the right-hand side (via the mapping $\psi_{\hat{h}}\left(u_{1}\right)$ ). Another difference is that for some technical reasons we would also like to ensure the existence of some ball around $\xi$ such that for every $\xi^{\prime}$ from this ball the same estimate on $\widetilde{N}_{\xi^{\prime}}$ as on $\widetilde{N}_{\xi}$ will hold. (The projection of $\tilde{h}$ on a finite-dimensional space to be defined later plays the role of $\xi$ here.) The third difference is due to the fact that we look for $\xi$ not in the ball of a prescribed small radius but in a convex subset of the ball. (This subset arises because the topology in the Euclidean space corresponds to $L^{2}$-topology in the functional space, and this subset corresponds to a set of functions close to zero in $C^{1}$ topology. We cannot derive any lower bounds of its volume and, so, need geometric Lemma 4.3 which makes possible to compare the volume of this set with a volume of a "forbidden" set corresponding to "bad" perturbations.) Nevertheless, one can cope with these additional features of the problem using the approach based on the quantitative Sard's theorem. As a result we get Lemma 4.4 which is the main ingredient in our proof of Theorems 1.1 and 1.2.

First consider a ball $B_{\rho}^{m}$ of radius $\rho>0$ in a Euclidean space $\mathbb{R}^{m}$. Let $\varphi: B_{\rho}^{m} \rightarrow \mathbb{R}^{m}$ be a $C^{2}$-smooth function. Let $R_{i}(\varphi)$ denote 


$$
\frac{1}{i !} \max _{y \in B_{\rho}^{m}}\left(\max _{0 \leq j \leq i}\left\|D^{j} \varphi(y)\right\|\right) \rho^{i} \quad(i \in\{1,2\}),
$$

$\Sigma(\varphi, \lambda)$ denote the set $\left\{x \in B_{\rho}^{m}\left|\min _{v \in \mathbb{R}^{m},|v|=1}\right| D \varphi(x) v \mid \leq \lambda\right\}$, and $\Delta(\varphi, \lambda)$ denote $\varphi(\Sigma(\varphi, \lambda))$.

The following theorem is a direct corollary of the quantitative Sard's theorem proven in [14].

Theorem 4.1 [14, 15]. There exists a universal constant $C_{0}>0$ such that for any positive $\varepsilon$ the minimal number of balls of radius $\varepsilon$ sufficient to cover the set $\Delta(\varphi, \lambda)$ (we denote this number by $M(\varphi, \lambda, \varepsilon)$ ) satisfies the following inequality:

$$
\begin{array}{r}
M(\varphi, \lambda, \varepsilon) \leq C_{0}^{m}\left[\sum_{q=0}^{m-1}\left(\frac{R_{1}(\varphi)}{\max \left\{R_{2}(\varphi)^{1 / 2} \varepsilon^{1 / 2}, \varepsilon\right\}}\right)^{q}\left(\max \left\{\frac{R_{2}(\varphi)}{\varepsilon}, 1\right\}\right)^{m / 2}\right. \\
\left.+\left(\frac{R_{1}(\varphi)}{\varepsilon}\right)^{m-1} \frac{\lambda \rho}{\varepsilon}\right] .
\end{array}
$$

Proof. Put $k=2, n=m$ and $\lambda_{1}=\cdots=\lambda_{n-1}=R_{1}(\varphi) / \rho, \lambda_{n}=\lambda$ in Theorem 9.2 in [15] (or Theorem 4.5 in [14]). Further, note that it follows from the proof of Theorem 9.2 in [15] (or of Theorem 4.5 in [14]) that the constant $C_{1}$ there grows with $m$ not quicker than exponentially when $n=m, k=2$ (in terms of Theorem 9.2 in [15]).

Remark. It is easy to verify that the right-hand side of (4.3) increases with $R_{1}(\varphi)$ and $R_{2}(\varphi)$. So, (4.3) will be true if we replace $R_{1}(\varphi), R_{2}(\varphi)$ by some upper bounds.

Lemma 4.2. Let $C, H>0$, and $K \in \mathbb{N}$ be fixed constants and for $i=1, \ldots, K$ $\varphi_{i}: B_{\rho}^{m} \rightarrow \mathbb{R}^{m}$ be $C^{2}$-smooth mappings. Let $R_{j}=\max _{i} R_{j}\left(\varphi_{i}\right) \quad(j \in\{1,2\})$. Suppose a positive $\gamma$ is given such that

$$
\gamma<R_{1} C_{0}
$$

(where $C_{0}$ is the same as in the text of Theorem 4.1).

Suppose that $K$ linear isometric embeddings $\chi_{i}: \mathbb{R}^{m} \rightarrow \mathbb{R}^{p}$ (where $m \leq$ $p \leq K m)$ are given and subspaces $\operatorname{Im} \chi_{i}(i \in\{1, \ldots, K\})$ span together $\mathbb{R}^{p}$. Denote by $\mu_{i}: \mathbb{R}^{p} \rightarrow \operatorname{Im} \chi_{i}$ orthogonal projections on these subspaces.

Further, we define $\varepsilon_{0}$ by the formula:

$$
\varepsilon_{0}=\min \left\{\frac{\gamma^{2 m} \cdot(\Gamma(m / 2+1))^{2}}{16 \cdot 9^{m} \cdot C_{0}^{2 m}(m / 2)^{m} R_{1}^{2 m-2} R_{2}(C+1)^{2} K^{m+2}}, R_{2}\right\},
$$

and $\delta^{*}$ by the formula

$$
\delta^{*}=\frac{\varepsilon_{0}}{H}
$$

Then there exists $\xi_{0} \in \mathbb{R}^{p}$ such that

(a) $\left|\xi_{0}\right| \leq \gamma$;

(b) Let $\varphi_{\varepsilon 1}, \varphi_{\varepsilon 2}, \ldots, \varphi_{\varepsilon K}$ be $K C^{2}$-smooth mappings $B_{\rho}^{m} \rightarrow \mathbb{R}^{m}$ such that for every $i \leq K \quad\left\|\varphi_{i}-\varphi_{\varepsilon i}\right\|_{C^{1}} \leq C \varepsilon_{0}$ and $\left\|D^{2} \varphi_{\varepsilon i}\right\| \leq H$. For any $i \leq K$ 
and for any $\xi \in \mathbb{R}^{p}$ such that $\left|\xi-\xi_{0}\right| \leq \varepsilon_{0}$ the cardinality of the inverse image set $\left(\chi_{i} \circ \varphi_{\varepsilon i}\right)^{-1}\left(\mu_{i}(\xi)\right)$ does not exceed $\left(2\left(\rho+\delta^{*}\right) / \delta^{*}\right)^{m}$, and a distance between any two elements of this set is not less than $\delta^{*}$.

Proof. We shall prove this lemma in two stages. First we shall prove that there exists $\xi_{0}$ satisfying (a) and such that for any $\xi$ satisfying $\left|\xi-\xi_{0}\right| \leq \varepsilon_{0}$ and for any mappings $\varphi_{\varepsilon i}$ satisfying the conditions of the lemma

$$
\operatorname{dist}\left(\mu_{i}(\xi), \chi\left(\Delta\left(\varphi_{\varepsilon i}, \varepsilon_{0}\right)\right)\right) \geq \varepsilon_{0},
$$

for any $i \in\{1, \ldots, K\}$. Secondly, we will prove that (4.6) implies (b).

To prove the existence of $\xi_{0}$ satisfying both (a) and inequality (4.6) we compare the volumes of the $p$-dimensional ball $B_{\gamma}^{p}$ of radius $\gamma$ centered at the origin and of the set $Q \in \mathbb{R}^{p}$, which we define as follows. We consider for every $i$ a system of $M\left(\varphi_{i},(C+1) \varepsilon_{0}, \varepsilon_{0}\right)$ balls of radius $\varepsilon_{0}$ in $\mathbb{R}^{m}$ covering $\Delta\left(\varphi_{i},(C+1) \varepsilon_{0}\right)$. Denote these balls by $B_{i j}$ (for every $i \in\{1, \ldots, K\} j$ changes from 1 to $\left.M\left(\varphi_{i},(C+1) \varepsilon_{0}, \varepsilon_{0}\right)\right)$. For every $B_{i j}$ we consider a concentric ball $B_{i j}^{\prime}$ in $\mathbb{R}^{m}$ of radius $3 \varepsilon_{0}$. Let $Q$ be $\left(\bigcup_{i, j} \mu_{i}^{-1}\left(\chi_{i}\left(B_{i j}^{\prime}\right)\right)\right) \cap B_{\gamma}^{p}$. Note that for every $i, j$,

$$
\operatorname{vol}\left(\mu_{i}^{-1}\left(\chi_{i}\left(B_{i j}^{\prime}\right)\right) \cap B_{\gamma}^{p}\right) \leq \begin{cases}3^{m} \varepsilon_{0}^{m} \omega_{m}, & \text { when } m=p, \\ 3^{m} \varepsilon_{0}^{m} \omega_{m} \gamma^{p-m} \omega_{p-m}, & \text { when } p>m,\end{cases}
$$

(here $\omega_{m}=2 \pi^{m / 2} /(m \Gamma(m / 2))$ denotes the volume of the unit $m$-dimensional ball, as before). Let $\omega_{0}=1$. Then for any $p \geq m$,

$$
\operatorname{vol}(Q) \leq 3^{m} \varepsilon_{0}^{m} \gamma^{p-m} \omega_{m} \omega_{p-m} K M\left(\varphi_{i},(C+1) \varepsilon_{0}, \varepsilon_{0}\right),
$$

and

$$
\operatorname{vol}\left(B_{\gamma}^{p}\right)=\gamma^{p} \omega_{p}
$$

Theorem 4.1 implies that

$$
M\left(\varphi_{i},(C+1) \varepsilon_{0}, \varepsilon_{0}\right) \leq 4 C_{0}^{m} \varepsilon_{0}^{-m+1 / 2} R_{1}^{m-1} \sqrt{R_{2}},
$$

if

$$
\varepsilon_{0} \leq \min \left\{\frac{R_{1}^{2}}{4 R_{2}}, \frac{4 R_{2}}{(C+1)^{2} \rho^{2}}, R_{2}\right\} .
$$

((4.10) is needed only to simplify the estimate for $M\left(\varphi_{i},(C+1) \varepsilon_{0}, \varepsilon_{0}\right)$.) It can be easily verified that (4.10) follows from (4.4), (4.5) and the obvious inequality $2 R_{2} \geq R_{1} \rho$. Substituting (4.9) and (4.5) into (4.7) we get

$$
\operatorname{vol}(Q) \leq \gamma^{p} \frac{\omega_{m} \omega_{p-m} \Gamma\left(\frac{m}{2}+1\right)}{\frac{m}{2}^{m / 2} K^{m / 2}} .
$$

But $K \geq p / m$ and it can be easily checked that for any $p \geq m \geq 1$,

$$
\frac{\Gamma\left(\frac{m}{2}+1\right) \omega_{m} \omega_{p-m}}{\left(\frac{m}{2}\right)^{m / 2}}\left(\frac{m}{p}\right)^{m / 2}<\omega_{p} .
$$

Now it follows from (4.8), (4.11) and (4.12) that $\operatorname{vol}(Q)<\operatorname{vol}\left(B_{\gamma}^{p}\right)$. Hence there exists a point $\xi_{0} \in B_{\gamma}^{p}$ such that for every $i \in\{1, \ldots, K\}$,

$$
\operatorname{dist}\left(\mu_{i}\left(\xi_{0}\right), \chi_{i}\left(\Delta\left(\varphi_{i},(C+1) \varepsilon_{0}\right)\right)\right) \geq 3 \varepsilon_{0}-\varepsilon_{0}=2 \varepsilon_{0} .
$$


So for any $\xi$ such that $\left|\xi-\xi_{0}\right| \leq \varepsilon_{0}$,

$$
\operatorname{dist}\left(\mu_{i}(\xi), \chi_{i}\left(\Delta\left(\varphi_{\varepsilon i}, \varepsilon_{0}\right)\right)\right) \geq \varepsilon_{0} .
$$

(Here we use the obvious fact that $\Delta\left(\varphi_{\varepsilon i}, \varepsilon_{0}\right) \subset \Delta\left(\varphi_{i},(C+1) \varepsilon_{0}\right)$.) This completes the first stage of the proof. Now our aim is to prove that (4.6) implies part (b) of the lemma.

Denote for convenience $\chi_{i} \circ \varphi_{\varepsilon i}$ by $q$ and $\mu_{i}(\xi)$ by $z_{0}$. Let $x_{0}$ be a solution of the equation $q(x)=z_{0}$. Consider the ball $B_{0}$ of radius $\delta^{*}$ around $x_{0}$ in $\mathbb{R}^{m}$. Set $D=B_{0} \cap B_{\rho}^{m}$. We should prove now that there are no other solutions in $D$. Let $x \in D$. Then

$$
\begin{aligned}
H\left|x-x_{0}\right|^{2} & \geq\left|q(x)-z_{0}-D q\left(x_{0}\right)\left(x-x_{0}\right)\right| \\
& =\left|D q\left(x_{0}\right)\left(x-x_{0}\right)-\left(q(x)-z_{0}\right)\right| \\
& \geq\left|D q\left(x_{0}\right)\left(x-x_{0}\right)\right|-\left|q(x)-z_{0}\right| .
\end{aligned}
$$

Thus,

$$
\left|q(x)-z_{0}\right| \geq\left|D q\left(x_{0}\right)\left(x-x_{0}\right)\right|-H\left|x-x_{0}\right|^{2} .
$$

But $\left(4.12^{\prime}\right)$ implies that $x_{0} \notin \Delta\left(q, \varepsilon_{0}\right)$. Thus,

$$
\begin{aligned}
\left|q(x)-z_{0}\right| & >\varepsilon_{0}\left|x-x_{0}\right|-H\left|x-x_{0}\right|^{2} \\
& \geq\left|x-x_{0}\right|\left(\varepsilon_{0}-H\left|x-x_{0}\right|\right) \geq 0 .
\end{aligned}
$$

So, $x_{0}$ is the unique solution of the equation $q(x)=z_{0}$ in $D$.

Hence the cardinality of the set $\left(\chi_{i} \circ \varphi_{\varepsilon i}\right)^{-1}\left(\mu_{i}(\xi)\right)$ does not exceed the maximal number of nonintersecting balls of diameter $\delta^{*}=\varepsilon_{0} / H$ with centers inside a ball of radius $\rho$. This completes the proof of the lemma.

We need the following purely geometrical lemma to compare volumes in a situation similar to the situation of the proof of Lemma 4.2 but in the case when instead of $B_{\gamma}^{p}$ we have a convex subset of it.

Lemma 4.3. Let $V$ be a compact convex body in the Euclidean space $\mathbb{R}^{n}$. Let $\mathbb{R}^{m}$ be any fixed linear subspace of $\mathbb{R}^{n}(0<m<n)$ and $\mathbb{R}^{n-m}$ be the orthogonal linear subspace. Denote by $p_{n-m}(V)$ a volume of the image of the orthogonal projection of $V$ on $\mathbb{R}^{n-m}$, and by $\operatorname{sect}_{m}(V)$ the maximal volume of a section by a m-dimensional linear submanifold parallel to the subspace $\mathbb{R}^{m}$, and by $\operatorname{vol}_{n}(V)$ the volume of $V$. Then

$$
\operatorname{vol}_{n}(V) \geq \frac{\operatorname{pr}_{n-m}(V) \operatorname{sect}_{m}(V)}{\left(\begin{array}{l}
n \\
m
\end{array}\right)} .
$$

Proof. First, we make the following observation. Suppose $A \subset \mathbb{R}^{m}, B \subset \mathbb{R}^{n-m}$ are convex sets such that $A \cap B=\{0\}$. Denote by $A * B$ the set $\{\lambda a+(1-\lambda) b \mid a \in$ $A ; b \in B ; \lambda \in[0,1]\}$. Then

$$
\operatorname{vol}_{n}(A * B)=\frac{\operatorname{vol}_{m}(A) \operatorname{vol}_{n-m}(B)}{\left(\begin{array}{l}
n \\
m
\end{array}\right)} .
$$

Indeed, if $A$ is the convex hull of the origin and $m$ vectors $a_{1}, \ldots, a_{m} \in \mathbb{R}^{m}$ and $B$ is the convex hull of the origin and $n-m$ vectors $b_{1}, \ldots, b_{n-m} \in \mathbb{R}^{n-m}$, then

$$
\begin{aligned}
\operatorname{vol}_{m}(A) & =\operatorname{det}\left(a_{1}, a_{2}, \ldots, a_{m}\right) / m ! \\
\operatorname{vol}_{n-m}(B) & =\operatorname{det}\left(b_{1}, \ldots, b_{n-m}\right) /(n-m) !
\end{aligned}
$$


and the orthogonality of $\mathbb{R}^{m}$ and $\mathbb{R}^{n-m}$ implies that

$$
\operatorname{vol}_{n}(A * B)=\operatorname{det}\left(a_{1}, \ldots, a_{m}\right) \operatorname{det}\left(b_{1}, \ldots, b_{n-m}\right) / n ! \text {. }
$$

Thus, the equality holds when $A$ and $B$ are simplices of the described above type with one vertice at the origin. But any convex set containing the origin can be approximated by disjoint unions of considered simplices. So, the equality is valid for all convex $A$ and $B$.

To prove the lemma we apply the symmetrization about $\mathbb{R}^{n-m}$ to $V$. We cross $V$ by all linear submanifolds $L^{m}$ parallel to $\mathbb{R}^{m}$. Let $B\left(L^{m}\right)$ be the ball in $L^{m}$ centered at $L^{m} \cap \mathbb{R}^{n-m}$ and of such radius that

$$
\operatorname{vol}_{m}\left(B\left(L^{m}\right)\right)=\operatorname{vol}_{m}\left(L^{m} \cap V\right) .
$$

Then by definition the result of the application of the symmetrization to $V$ will be $\bigcup_{L^{m}} B\left(L^{m}\right)$. We denote it by $S V$. It is well known that $S V$ will be a compact convex body and $\operatorname{vol}_{n}(S V)=\operatorname{vol}_{n}(V)$ (cf. [21]). Note that $\operatorname{sect}_{m}(S V)=$ $\operatorname{sect}_{m}(V)$ and that $S V \cap \mathbb{R}^{n-m}$ is a convex set such that $\operatorname{vol}_{n-m}\left(S V \cap \mathbb{R}^{n-m}\right)=$ $\operatorname{pr}_{n-m}(V)$. Suppose that $\operatorname{sect}_{m}(V)=\operatorname{vol}_{m}\left(V \cap L_{0}^{m}\right)$ for some linear submanifold $L_{0}^{m}$ parallel to $\mathbb{R}^{m}$. We may consider the point $\left(S V \cap L_{0}^{m}\right) \cap\left(S V \cap \mathbb{R}^{n-m}\right)$ as the new origin. Note that the set $\left(S V \cap L_{0}^{m}\right) *\left(S V \cap \mathbb{R}^{n-m}\right)$ is contained in $S V$ because of the convexity of $S V$. By the observation at the beginning of the proof

$$
\begin{aligned}
\operatorname{vol}_{n}(V) & =\operatorname{vol}_{n}(S V) \geq \operatorname{vol}_{n}\left(\left(S V \cap L_{0}^{m}\right) *\left(S V \cap \mathbb{R}^{n-m}\right)\right) \\
& =\frac{\operatorname{vol}_{m}\left(S V \cap L_{0}^{m}\right) \operatorname{vol}_{n-m}\left(S V \cap \mathbb{R}^{n-m}\right)}{\left(\begin{array}{c}
n \\
m
\end{array}\right)} \\
& =\frac{\operatorname{sect}_{m}(V) \operatorname{pr}_{n-m}(V)}{\left(\begin{array}{c}
n \\
m
\end{array}\right)} .
\end{aligned}
$$

In Lemma 4.2 we dealt with mappings $\chi_{i} \circ \varphi_{i}\left(\right.$ or $\left.\chi_{i} \circ \varphi_{\varepsilon i}\right)$ from $B_{\rho}^{m}$ to an $m$ dimensional subspace of $\mathbb{R}^{k}$ (denote this subspace $\mathbb{R}_{i}^{m}$ ). Now we would like to prove the similar result but for the case of mappings $\psi_{i} \equiv \chi \circ \varphi_{i}: B_{\rho}^{m_{i}} \times\left(\mathbb{R}_{i}^{m_{i}}\right)^{\perp} \rightarrow$ $\mathbb{R}_{i}^{m_{i}} \subset \mathbb{R}^{p}$. $\left(\mathbb{R}_{i}^{m_{i}}\right)^{\perp}$ denotes the orthogonal complement from $\mathbb{R}_{i}^{m_{i}}$ to $\mathbb{R}^{p}$. Note that the dimension $m_{i}$ of the domain depends now on $i$, but we assume that for any $i, m_{i} \leq m$. Even more important difference is that now we look for the point $\xi$ not in the ball of radius $\gamma$ but in a convex subset $V_{\gamma}$ of this ball.

Lemma 4.4. Let $C, \gamma, H>0$, and $m, p, K \in \mathbb{N}$ be fixed constants such that $m \leq p \leq m K$, and for $i=1, \ldots, K$ let $\mathbb{R}_{i}^{m_{i}}$ be $m_{i}$-dimensional linear subspaces of $\mathbb{R}^{p}$, where $m_{i} \leq m$. Let, for $i \in\{1, \ldots, K\}, \psi_{i}: B_{\rho}^{m_{i}} \times\left(\mathbb{R}_{i}^{m_{i}}\right)^{\perp} \rightarrow$ $\mathbb{R}_{i}^{m_{i}}$ be $C^{2}$-mappings, and for every $y \in\left(\mathbb{R}_{i}^{m_{i}}\right)^{\perp}, \psi_{i y}$ denotes the mapping $\psi_{i}(\cdot, y): B_{\rho}^{m_{i}} \rightarrow \mathbb{R}_{i}^{m_{i}}$. Let $R_{j}=\max _{i} \sup _{y \in B_{m_{i}}^{\rho}} R_{j}\left(\psi_{i y}\right)$ (where $B_{\gamma}^{p}$ is the ball of the radius $\gamma<\rho$ in $\mathbb{R}^{p}$ centered at the origin and $R_{j}\left(\psi_{i y}\right)$ is defined as before the text of Lemma 4.2).

Denote by $\mu_{i}$ linear orthogonal projections from $\mathbb{R}^{p}$ on $\mathbb{R}_{i}^{m_{i}}$ and by $\mu_{i}^{\perp}$ linear orthogonal projections from $\mathbb{R}^{p}$ on $\left(\mathbb{R}_{i}^{m_{i}}\right)^{\perp}$. Assume that $\gamma<R_{1} C_{0}$, where $C_{0}$ is defined in the text of Theorem 4.1, and in addition $\gamma \leq 1$. Suppose that $\varepsilon_{0}$ is defined by the formula

$$
\varepsilon_{0}=\min \left\{\frac{\gamma^{2 m}}{16\left(\max \left\{3 C_{0}, 1\right\}\right)^{2 m} R_{1}^{2 m-2} R_{2} K^{2 m+2} m^{2 m}(C+1)^{2}}, R_{2}\right\},
$$


and $\delta^{*}$ is defined by the formula

$$
\delta^{*}=\frac{\varepsilon_{0}}{H}
$$

Consider closed balls $B_{\gamma}^{m_{i}}(0)$ of radius $\gamma$ centered at the origin in all subspaces $\mathbb{R}_{i}^{m_{i}}$. Define a set $V_{\gamma}$ as a convex hull of all these sets $B_{\gamma}^{m_{i}}(0)$. (Thus

$$
\left.V_{\gamma}=\left\{\lambda_{1} x_{1}+\cdots+\lambda_{k} x_{k}\left|\sum_{i=1}^{K} \lambda_{i}=1, x_{i} \in \mathbb{R}_{i}^{m_{i}},\right| x_{i} \mid \leq \gamma\right\} .\right)
$$

Then there exists $\xi_{0} \in \mathbb{R}^{p}$ such that

(a) $\xi_{0} \in V_{\gamma}$;

(b) Let, for each $i \in\{1, \ldots, K\}, \quad \psi_{\varepsilon i}: B_{\rho}^{m_{i}} \times\left(\mathbb{R}_{i}^{m_{i}}\right)^{\perp} \rightarrow \mathbb{R}^{m_{i}}$ be a $C^{2}$ smooth mapping such that $\left\|\psi_{i}-\psi_{\varepsilon i}\right\|_{C^{1}} \leq C \varepsilon_{0}$ and $\left\|D^{2} \psi_{\varepsilon i}\right\| \leq H$. For any $i \in\{1, \ldots, K\}$ and for any $\xi \in \mathbb{R}^{p}$ such that $\left|\xi-\xi_{0}\right| \leq \varepsilon_{0}$ the cardinality of the inverse image set $\psi_{\varepsilon i \mu_{i}^{\perp}(\xi)}^{-1}\left(\mu_{i}(\xi)\right)$ does not exceed $\left(2\left(\rho+\delta^{*}\right) / \delta^{*}\right)^{m}$ and a distance between any two elements of this set is not less than $\delta^{*}$. Here $\psi_{\varepsilon i \mu_{i}^{\perp}(\xi)}$ denotes the mapping $\psi_{\varepsilon i}\left(\cdot, \mu_{i}^{\perp}(\xi)\right): B_{\rho}^{m_{i}} \rightarrow \mathbb{R}_{i}^{m_{i}}$.

Proof. It follows the proof of Lemma 4.2. First, we would like to prove that there exists $\xi_{0}$ satisfying (a) and such that for any $\xi$ satisfying $\left|\xi-\xi_{0}\right| \leq \varepsilon_{0}$,

$$
\operatorname{dist}\left(\mu_{i}(\xi), \Delta\left(\psi_{\varepsilon i \mu_{i}^{\perp}(\xi)}, \varepsilon_{0}\right)\right) \geq \varepsilon_{0} .
$$

Then the proof can be completed exactly as the proof of Lemma 4.2.

To prove the existence of such $\xi_{0}$ we make a comparison of volumes as in the proof of Lemma 4.2. Namely, we compare the volume of $V_{\gamma}$ with the volume of the set $\widetilde{Q} \in \mathbb{R}^{p}$ defined as follows. For every $\xi \in B_{\gamma}^{p}$ and every $i \in\{1, \ldots, K\}$ let $\mathbb{R}_{i}^{m_{i}}(\xi)$ denote the linear submanifold $\mathbb{R}_{i}^{m_{i}}+\mu_{i}^{\perp}(\xi)$ passing through $\xi$ and parallel to $\mathbb{R}_{i}^{m_{i}}$. Define also mappings $\tilde{\psi}_{i \xi}: B_{\rho}^{m_{i}} \rightarrow \mathbb{R}_{i}^{m_{i}}(\xi)$,

$$
\tilde{\psi}_{i \xi}(x)=\psi_{i}\left(x, \mu_{i}^{\perp}(\xi)\right)+\mu_{i}^{\perp}(\xi) .
$$

By Theorem 4.1 there exists a cover $\operatorname{COV}(\xi)$ of $\Delta\left(\tilde{\psi}_{i \xi},(C+1) \varepsilon_{0}\right)$ by not more than

$$
\begin{aligned}
& C_{0}^{m_{i}}\left(\sum_{q=0}^{m_{i}-1}\left(\frac{R_{1}\left(\psi_{i \mu_{i}^{\perp}(\xi)}\right)}{R_{2}\left(\psi_{i \mu_{i}^{\perp}(\xi)}\right)^{1 / 2} \varepsilon_{0}^{1 / 2}}\right)^{q}\left(\frac{R_{2}\left(\psi_{i \mu_{i}^{\perp}(\xi)}\right)}{\varepsilon_{0}}\right)^{m_{i} / 2}\right. \\
&\left.+(C+1) \rho\left(\frac{R_{1}\left(\psi_{i \mu_{i}^{\perp}(\xi)}\right)}{\varepsilon_{0}}\right)^{m_{i}-1}\right)
\end{aligned}
$$

balls of radius $\varepsilon_{0}$ (here we used the obvious fact that $R_{1}\left(\tilde{\psi}_{i \xi}\right)=R_{1}\left(\psi_{i \mu_{i}^{\perp}(\xi)}\right)$ and $R_{2}\left(\tilde{\psi}_{i \xi}\right)=R_{2}\left(\psi_{i \mu_{i}^{\perp}(\xi)}\right)$. For every ball $B \in \operatorname{COV}(\xi)$ we consider a concentric ball $B_{3}(B)$ of radius $3 \varepsilon_{0}$. Define $\widetilde{Q}_{i}(\xi) \subset \mathbb{R}_{i}^{m_{i}}(\xi)$ as $\left(\bigcup_{B \in \operatorname{COV}(\xi)} B_{3}(B)\right) \cap V_{\gamma}$. Note that $\widetilde{Q}_{i}(\xi)$ depends only on $\mu_{i}^{\perp}(\xi)$. Define $\widetilde{Q}_{i}$ by

$$
\tilde{Q}_{i}=\bigcup_{\xi \in B_{\gamma}^{p}} \tilde{Q}_{i}(\xi)=\bigcup_{\xi \in B_{\gamma}^{p} \cap\left(\mathbb{R}_{i}^{m_{i}}\right)^{\perp}} \tilde{Q}_{i}(\xi)=\bigcup_{\xi \in \mu_{i}^{\perp}\left(V_{\gamma}\right)} \widetilde{Q}_{i}(\xi),
$$


and let

$$
\widetilde{Q}=\bigcup_{i=1}^{K} \widetilde{Q}_{i}
$$

We want to prove that under the assumptions of Lemma $4.4 \operatorname{vol}(\widetilde{Q})<$ $\operatorname{vol}\left(V_{\gamma}\right)$. For every $i \leq K$ and every $\xi \in\left(\mathbb{R}_{i}^{m_{i}}\right)^{\perp}$,

$$
\begin{aligned}
\operatorname{vol}_{m_{i}}\left(\widetilde{Q}_{i} \cap \mathbb{R}_{i}^{m_{i}}(\xi)\right) \leq C_{0}^{m_{i}}\left(\sum_{q=0}^{m_{i}-1}\left(\frac{R_{1}\left(\psi_{i \xi}\right)}{R_{2}\left(\psi_{i \xi}\right)^{1 / 2} \varepsilon_{0}^{1 / 2}}\right)^{q}\left(\frac{R_{2}\left(\psi_{i \xi}\right)}{\varepsilon_{0}}\right)^{m_{i} / 2}\right. \\
\left.+(C+1) \rho\left(\frac{R_{1}\left(\psi_{i \xi}\right)}{\varepsilon_{0}}\right)^{m_{i}-1}\right) \cdot\left(3 \varepsilon_{0}\right)^{m_{i}} \omega_{m_{i}} \\
\leq C_{0}^{m_{i}}\left(\sum_{q=0}^{m_{i}-1}\left(\frac{R_{1}}{R_{2}^{1 / 2} \varepsilon_{0}^{1 / 2}}\right)^{q}\left(\frac{R_{2}}{\varepsilon_{0}}\right)^{m_{i} / 2}+(C+1) \rho\left(\frac{R_{1}}{\varepsilon_{0}}\right)^{m_{i}-1}\right)\left(\left(3 \varepsilon_{0}\right)^{m_{i}} \omega_{m_{i}}\right) .
\end{aligned}
$$

By Fubini's theorem

$$
\begin{aligned}
\operatorname{vol}\left(\widetilde{Q}_{i}\right) \leq C_{0}^{m_{i}}\left(\sum_{q=0}^{m_{i}-1}\left(\frac{R_{1}}{R_{2}^{1 / 2} \varepsilon_{0}^{1 / 2}}\right)^{q}\left(\frac{R_{2}}{\varepsilon_{0}}\right)^{m_{i} / 2}\right. \\
\left.+(C+1) \rho\left(\frac{R_{1}}{\varepsilon_{0}}\right)^{m_{i}-1}\right)\left(3 \varepsilon_{0}\right)^{m_{i}} \omega_{m_{i}} \operatorname{pr}_{p-m_{i}}^{\perp}\left(V_{\gamma}\right),
\end{aligned}
$$

where $\operatorname{pr}_{p-m_{i}}^{\perp}\left(V_{\gamma}\right)$ denotes the volume of $\mu_{i}^{\perp}\left(V_{\gamma}\right)$ in $\left(\mathbb{R}_{i}^{m_{i}}\right)^{\perp}$.

Under the assumption $\gamma<R_{1} C_{0}$ and from the inequality (4.10) which follows from (4.13) we get as in the proof of Lemma 4.2. that

$$
\begin{aligned}
& \sum_{q=0}^{m_{i}-1}\left(\frac{R_{1}}{R_{2}^{1 / 2} \varepsilon_{0}^{1 / 2}}\right)^{q}\left(\frac{R_{2}}{\varepsilon_{0}}\right)^{m_{i} / 2}+(C+1) \rho\left(\frac{R_{1}}{\varepsilon_{0}}\right)^{m_{i}-1} \\
& \quad \leq 4 \varepsilon_{0}^{-m_{i}+1 / 2} R_{1}^{m_{i}-1} R_{2}^{1 / 2} .
\end{aligned}
$$

Hence

$$
\operatorname{vol}\left(\widetilde{Q}_{i}\right) \leq 4 C_{0}^{m_{i}} \varepsilon_{0}^{-m_{i}+1 / 2} R_{1}^{m_{i}-1} \sqrt{R_{2}}\left[\left(3 \varepsilon_{0}\right)^{m_{i}} \omega_{m_{i}} \operatorname{pr}_{p-m_{i}}^{\perp}\left(V_{\gamma}\right)\right]
$$

By Lemma 4.3

$$
\operatorname{vol}\left(V_{\gamma}\right) \geq \frac{\omega_{m_{i}} \gamma^{m_{i}} \operatorname{pr}_{p-m_{i}}^{\perp}\left(V_{\gamma}\right)}{\left(\begin{array}{c}
p \\
m_{i}
\end{array}\right)}
$$

Hence

$$
\frac{\operatorname{vol}\left(\tilde{Q}_{i}\right)}{\operatorname{vol}\left(V_{\gamma}\right)} \leq 4\left(3 C_{0}\right)^{m_{i}} \varepsilon_{0}^{1 / 2} R_{1}^{m_{i}-1} R_{2}^{1 / 2} \gamma^{-m_{i}}\left(\begin{array}{c}
p \\
m_{i}
\end{array}\right) .
$$

Note that $\left(\begin{array}{c}p \\ m_{i}\end{array}\right) \leq(K m)^{m_{i}}$. Using the assumption $\gamma \leq 1$ we see that

$$
\frac{\sum_{i=1}^{K} \operatorname{vol}\left(\tilde{Q}_{i}\right)}{\operatorname{vol}\left(V_{\gamma}\right)} \leq 4\left(3 C_{0}\right)^{m} \varepsilon_{0}^{1 / 2} m^{m} K^{m+1} R_{1}^{m-1} \sqrt{R_{2}} \gamma^{-m} \text {. }
$$


Now (4.19) and (4.13) imply that $\operatorname{vol}(\widetilde{Q})<\operatorname{vol}\left(V_{\gamma}\right)$. Hence there exists a point $\xi_{0} \in V_{\gamma} \backslash \widetilde{Q}$ and for this point for every $i \in\{1, \ldots, K\}$,

$$
\operatorname{dist}\left(\mu_{i}\left(\xi_{0}\right), \Delta\left(\psi_{\varepsilon i \mu_{i}^{\perp}\left(\xi_{0}\right)}, \varepsilon_{0}\right)\right) \geq 3 \varepsilon_{0}-\varepsilon_{0}=2 \varepsilon_{0} .
$$

This implies (4.14) for all $\xi$ such that $\left|\xi-\xi_{0}\right| \leq \varepsilon_{0}$. From this point the proof continues exactly as the proof of Lemma 4.2.

\section{Proof of MAIN Results}

To prove Theorem 1.1 and Theorern 1.2, we proceed as follows. Let $\rho(S)=$ $r(S+r(S))$, where $r(S)$ is defined by (3.23), (3.21), (3.46). Consider the cover of the solution set of all problems (1.3) with $h$ satisfying (1.2) by balls $B$ such that the radius $(B) \leq \rho\left(\|\right.$ the center $\left.(B) \|_{C_{0}(\bar{\Omega})}\right)$, the existence of which is stated in Proposition 2.4. An upper bound on the number of balls in such a cover is given by Proposition 2.4(a). We can see immediately that $\rho(S)$ satisfies the inequality

$$
\rho(S) \leq \bar{C}_{8}(N,|\Omega|) \bar{M}_{2}\left(S+\frac{C_{8}(N,|\Omega|)}{\bar{M}_{2}(S)^{7 N / 4}}\right)^{-7 N / 4},
$$

where the constants $C_{8}(N,|\Omega|)$ and $\bar{C}_{8}(N,|\Omega|)$ were defined by the inequalities (3.49) and (3.50). Results of $\S 3$ permit reduction of the local problems concerning numbers of solutions in small balls $B$ of the cover to finite dimensional problems, and results of $\S 4$ allow us to prove the existence of $C^{\alpha}$-smooth $h$ with arbitrary small $C^{0}$-norm such that all these local problems do not have very many solutions. Moreover, this property persists under small perturbations of $h$. When we will apply results of $\S 3$ we take $S+r(S)$ and not $S$ as a value of the a priori upper bound on the $C^{0}$-norm of solutions. We do so because otherwise small balls centered at points $u_{0}$ with $C^{0}$-norms almost equal to $S$ will be partially outside the ball $\left\{u \in C^{\infty}(\bar{\Omega}) \mid\|u\|_{C^{0}(\bar{\Omega})} \leq S\right\}$. Thus, all estimates will be in the terms of $\bar{M}_{2}(S+r(S))$. But the assumption that for any $c>0, \bar{M}_{2}\left(S+c / \bar{M}_{2}(S)\right)=\bar{M}_{2}(S)(1+o(1))$ when $S \rightarrow+\infty$, makes it possible to write all estimates in terms of $\bar{M}_{2}(S)$. Thus, we get Theorem 1.1 .

To prove Theorem 1.2 we use the freedom to perturb $h$ a bit while not increasing significantly the number of solutions with $C^{0}$-norm $\leq S$. We construct the sequence $\left\{S_{i}\right\}_{i=1}^{\infty}$ such that $\lim _{i \rightarrow \infty} S_{i}=+\infty$ and a sequence $\tilde{h}_{i}-h_{0}$ of $C^{2+\alpha}$ perturbations of the right-hand side $h_{0}$ of the problem converging to a $C^{1}$-smooth limit $h_{\infty}-h_{0}$ such that the number of solutions $u$ of the problem

$$
\left\{\begin{array}{l}
-\Delta u+f(u, x)=h_{\infty} \\
\left.u\right|_{\partial \Omega}=0
\end{array}\right.
$$

such that $\|u\|_{C^{0}(\bar{\Omega})} \leq S_{i}$ satisfies (1.9).

Proof of Theorem 1.1. Let $\bar{S}=S+r(S)$, where $r(S)$ is defined by (3.23), (3.21), (3.46). Now choose a new value of $\varepsilon$. We put $\varepsilon^{\prime}=\min \{\varepsilon, \delta(\bar{S}) / 2\}$, where the function $\delta$ is defined by formulae (3.46), (3.21), (3.22), and satisfies (3.48). Subtracting $h_{0}$ from $f(u, x)$ we reduce the theorem to the case $h_{0}(x) \equiv 0$. Consider a cover of the set of a priori solutions $u$ such that $\|u\|_{C^{0}(\bar{\Omega})} \leq S$ of all boundary value problems (1.3) with $C^{\alpha}$-smooth right-hand sides $h$ such that 
$\|h\|_{C^{0}(\bar{\Omega})} \leq \varepsilon^{\prime}$ by $C^{0}$-norm balls of radius $\rho(S)=r(S+r(S))$. This cover can be chosen to satisfy statements (a) and (b) of Proposition 2.4. So, we may assume that the number of balls $N B(S)$ in the cover satisfies the following inequality:

$$
\ln N B(S) \leq C_{18}(N, \operatorname{diam} \Omega)\left((\operatorname{dil} \Omega)^{N} S^{N} \bar{M}_{0}^{N}(S) \bar{M}_{2}(\bar{S})^{7 N^{2} / 4}\right),
$$

where $C_{18}(N, \operatorname{diam} \Omega)$ is a constant which can be explicitly written.

Note that for any $u_{0}$ such that $\left\|u_{0}\right\|_{C^{0}(\bar{\Omega})} \leq S, \rho(S) \leq \bar{S}-\left\|u_{0}\right\|_{C^{0}(\bar{\Omega})}$. By results of $\S 3$ the number of solutions of a problem (1.3) with a right-hand side $\tilde{h}$ satisfying $\|\tilde{h}\|_{C^{0}(\bar{\Omega})} \leq \varepsilon^{\prime}$ belonging to a ball $B$ of the cover does not exceed the number of solutions of the equation $T_{\tilde{h}}^{\left(u_{B}\right)}\left(u_{1}\right)=0$. Here the mapping $T_{\hat{h}}^{\left(u_{B}\right)}$ is defined as $T_{\tilde{h}}$ in $\S 3$ for the center $u_{B}$ of the ball $B$ as $u_{0}$. The mapping $T_{\tilde{h}}^{\left(u_{B}\right)}$ is defined on a ball $B$ of radius $r(\bar{S})$ in the space $P_{1}\left(u_{B}\right)$ determined by $u_{B}$ exactly as $P_{1}$ was determined by $u_{0}$ in $\S 3$. This ball is mapped into the space $Q_{1}\left(u_{B}\right)$ determined by $u_{B}$ exactly as $Q_{1}$ was by $u_{0}$. More precisely, $P_{1}\left(u_{B}\right)=\operatorname{span}\left\{v_{i}(X) \mid v_{i}(X)\right.$ is an eigenfunction corresponding to an eigenvalue $\lambda_{i}$ of the operator $-\Delta+f_{u}^{\prime}\left(u_{B}(x), x\right)$ such that $\left.\lambda_{i} \leq a(\bar{S})+b(\bar{S})\right\}$, where $a(\bar{S})$ and $b(\bar{S})$ are defined by (3.46),

$$
\begin{aligned}
Q_{1}\left(u_{B}\right) & =\operatorname{Coker}\left(-\Delta+f_{u}^{\prime}\left(u_{B}(x), x\right)\right) \oplus \operatorname{Im} P_{1}\left(u_{B}\right)=P_{1}\left(u_{B}\right), \\
P_{2}\left(u_{B}\right) & =P_{1}^{\perp}\left(u_{B}\right), \quad Q_{2}\left(u_{B}\right)=Q_{1}^{\perp}\left(u_{B}\right), \\
T_{\grave{h}}^{\left(u_{B}\right)}\left(u_{1}\right) & =\pi_{Q_{1}\left(u_{B}\right)} F\left(u_{1}, \psi_{\hat{h}}^{\left(u_{B}\right)}\left(u_{1}\right)\right)-\pi_{Q_{1}\left(u_{B}\right)} \tilde{h},
\end{aligned}
$$

where $\psi_{\hat{h}}^{\left(u_{B}\right)}$ is also defined exactly as in $\S 3$ (from equation (3.24)). We suppose that all considered spaces are equipped with the norm of $L^{2}(\bar{\Omega})$. Thus, they are isomorphic to Euclidean spaces. Denote $\pi_{Q_{1}\left(u_{B}\right)} F\left(u_{1}, \psi_{\hat{h}}^{\left(u_{B}\right)}\left(u_{1}\right)\right)$ by $\bar{T}_{\grave{h}}^{\left(u_{B}\right)}\left(u_{1}\right)$. Note that $D \bar{T}_{\hat{h}}^{\left(u_{B}\right)}\left(u_{1}\right)=D T_{\grave{h}}^{\left(u_{B}\right)}\left(u_{1}\right)$. Hence Proposition 3.4 also describes properties of $\bar{T}_{\hat{h}}^{\left(u_{B}\right)}$ (only (c) holds with another constant). Thus, the choice of $a(\bar{S}), b(\bar{S})$ by (3.46) implies the estimates (3.52)-(3.55) but on $\bar{T}_{\hat{h}}^{\left(u_{B}\right)}$ instead of $T_{\hat{h}}^{\left(u_{B}\right)}$. The equation $T_{\tilde{h}}^{\left(u_{B}\right)}\left(u_{1}\right)=0$ is equivalent to the equation $\bar{T}_{\tilde{h}}^{\left(u_{B}\right)}\left(u_{1}\right)=\pi_{Q_{1}\left(u_{B}\right)} \tilde{h}$. Note that by its definition $\bar{T}_{\tilde{h}}^{\left(u_{B}\right)}$ depends on $\pi_{Q_{2}\left(u_{B}\right)} \tilde{h}$ but not on $\pi_{Q_{1}\left(u_{B}\right)} \tilde{h}$.

Now we apply Lemma 4.4. We regard spaces $Q_{1}\left(u_{B_{i}}\right)$ as $\mathbb{R}_{i}^{m_{i}}$. Denote their sum (not direct in general) by $\bar{Q}_{1}$. It is regarded as $\mathbb{R}^{p}$. For $\tilde{h} \in \bar{Q}_{1}$ the mappings $\bar{T}_{\tilde{h}}^{\left(u_{B_{i}}\right)}\left(u_{1}\right) \quad(i \in\{1, \ldots, N B(S)\})$ are regarded as $\psi_{i}\left(u_{1}, \pi_{Q_{2}\left(B_{i}\right)} \tilde{h}\right)$ (in the notations of Lemma 4.4). Let

$$
m_{i}=\operatorname{dim} P_{1}\left(u_{B_{i}}\right), \quad K=N B(S) .
$$

The value of $\rho$ coincides with $r(\bar{S})$ which satisfies (3.23), (3.49) and

$$
\gamma=\min \left\{\frac{\varepsilon^{\prime}}{\operatorname{const}(N, \operatorname{diam} \Omega) \bar{M}_{2}(S)^{3 N / 4+1}}, R_{1} C_{0}\right\} .
$$

Here $C_{0}$ is the constant defined in of Theorem 4.1 and $\operatorname{const}(N$, diam $\Omega)$ will be defined later. The estimates for $R_{1}, R_{2}$ (in the sense of Lemma 4.4) are provided by (3.52), (3.53), and (3.49). We can regard $\bar{T}_{g}^{\left(u_{B_{i}}\right)}\left(u_{1}\right)$ for such $g$ 
that $\|g-h\|_{C^{0}(\bar{\Omega})} \leq \nu(S, \varepsilon)$ as $\psi_{\varepsilon i}$. The estimates for $H$ and for $C$ are provided by (3.53), (3.54), and (3.55). The value of $\xi_{0}$ existence of which is stated in Lemma 4.4 considered as a function on $\bar{\Omega}$ will be the necessary $h$ and we can put $\nu(S, \varepsilon)$ to be equal to $\delta^{*}$ defined by the formula $\left(4.13^{\prime}\right)$. The application of Lemma 4.4 proves immediately parts (2), (3) of Theorem 1.1.

The function $h \in C^{2+\alpha}(\bar{\Omega})$ because it is a linear combination of a finite number of eigenfunctions of the operators $-\Delta+f_{u}^{\prime}\left(u_{B_{i}}(x), x\right)$ and $u_{B_{i}} \in C^{2+\alpha}(\bar{\Omega})$. To prove that $\|h\|_{C^{1}(\bar{\Omega})} \leq \varepsilon$ we proceed as follows. By Lemma 4.4

$$
h \equiv \xi_{0}=\sum_{i=1}^{K} \lambda_{i} v_{B_{i}}
$$

where $v_{B_{i}}$ is a linear combination of eigenfunctions of the operator $-\Delta+$ $f_{u}^{\prime}\left(u_{B_{i}}(x), x\right)$ satisfying

$$
\left\|v_{B_{i}}\right\|_{L^{2}(\bar{\Omega})} \leq \frac{\varepsilon^{\prime}}{\operatorname{const}(N, \operatorname{diam} \Omega) \bar{M}_{2}(S)^{3 N / 4+1}}, \quad \lambda_{i} \geq 0,
$$

and $\sum_{i=1}^{K} \lambda_{i} \leq 1$. Hence, by Proposition 3.1(4) and Proposition 3.1(1) we can choose $\operatorname{const}(N, \operatorname{diam} \Omega)$ such that

$$
\|h\|_{C^{1}(\bar{\Omega})} \leq \sum_{i=1}^{K} \lambda_{i}\left\|v_{B_{i}}\right\|_{C^{1}(\bar{\Omega})} \leq \max _{i}\left\|v_{B_{i}}\right\|_{C^{1}(\bar{\Omega})} \leq \varepsilon .
$$

Proof of Theorem 1.2. Let us introduce a function $\tilde{\nu}(S, \varepsilon)$ by the formula

$$
\begin{aligned}
\tilde{\nu}(S, \varepsilon)=\exp \left(-\Theta_{2}(N, \operatorname{diam} \Omega)(\right. & (\operatorname{dil} \Omega)^{N} S^{N}\left(\bar{M}_{0}(S)+\|h\|_{C^{0}(\bar{\Omega})}\right)^{N} \\
& \left.\left.\times\left(\bar{M}_{2}(S)\right)^{7 N^{2} / 4+N / 2}+\bar{M}_{2}(S)^{N / 2} \ln 1 / \varepsilon\right)\right) .
\end{aligned}
$$

Thus, (1.8) can be written as $\nu(S, \varepsilon) \geq \tilde{\nu}(S, \varepsilon)$.

Consider now an increasing sequence $\left\{S_{i}\right\}_{i=1}^{\infty}$ such that $\lim _{i \rightarrow+\infty} S_{i}=+\infty$. As in the proof of Theorem 1.1 we take a new value of $\varepsilon$. Namely, let $\varepsilon_{1} \leq$ $\min \left\{\varepsilon / 4, \delta\left(S_{1}\right) / 4\right\}$ be a positive constant such that for any positive $\tilde{\varepsilon} \leq \varepsilon_{1}$ and any $S \geq S_{1}, \tilde{\nu}(S, \tilde{\varepsilon})<\tilde{\varepsilon}$. Here and below $\delta(S)$ is the function defined in $\S 3$ and satisfying (3.48).

Now we apply Theorem 1.1. The theorem implies that there exists a $C^{2+\alpha_{-}}$ smooth function (we denote it by $h_{1}$ ) such that (1) $\left\|h_{1}\right\|_{C^{1}(\bar{\Omega})} \leq \varepsilon_{1}$; (2) The number $N_{g}\left(S_{1}\right)$ of $C^{2+\alpha}$-smooth solutions $u$ such that $\|u\|_{C^{0}(\bar{\Omega})} \leq S_{1}$ of the problem

$$
\left\{\begin{array}{l}
-\Delta u+f(u, x)-h_{0}(x)=g(x) \quad \text { in } \Omega \\
\left.u\right|_{\partial \Omega}=0
\end{array}\right.
$$

for any $C^{\alpha}$-smooth function $g$ such that $\left\|g-h_{1}\right\|_{C^{0}(\bar{\Omega})} \leq \tilde{\nu}\left(S_{1}, \varepsilon_{1}\right)$ satisfies inequality (1.7) for $S=S_{1}$.

Proceeding by induction we set for every $j \geq 1$,

$$
\varepsilon_{j+1}=\min \left\{\frac{\tilde{\nu}\left(S_{j}, \varepsilon_{j}\right)}{4}, \frac{\delta\left(S_{j+1}\right)}{4}\right\} .
$$

Then we apply Theorem 1.1 to the boundary value problem

$$
\left\{\begin{array}{l}
-\Delta u+\left(f(u, x)-h_{0}(x)-h_{1}(x)-\cdots-h_{j}(x)\right)=0 \quad \text { in } \Omega \\
\left.u\right|_{\partial \Omega}=0
\end{array}\right.
$$


As a result of the application of the theorem we get the existence of a $C^{2+\alpha}$ smooth function $h_{j+1}$ such that:

(1) the number $N_{g}\left(S_{j+1}\right)$ of $C^{2+\alpha}$-smooth solutions $u$ such that $\|u\|_{C^{0}(\bar{\Omega})} \leq$ $S_{j+1}$ of the boundary value problem

$$
\left\{\begin{array}{l}
-\Delta u+\left(f(u, x)-h_{0}(x)-h_{1}(x)-\cdots-h_{j}(x)\right)=g(x) \quad \text { in } \Omega, \\
\left.u\right|_{\partial \Omega}=0
\end{array}\right.
$$

satisfies the inequality (1.7) for $S=S_{j+1}$ and with $\sum_{i=0}^{j} h_{i}$ instead of $h_{0}$ when $g \in C^{\alpha}(\bar{\Omega})$ and $\left\|g-h_{j+1}\right\|_{C^{0}(\bar{\Omega})} \leq \tilde{\nu}\left(S_{j+1}, \varepsilon_{j+1}\right)$;

(2) $\left\|h_{j+1}\right\|_{C^{1}(\bar{\Omega})} \leq \varepsilon_{j+1}$.

For any $j \geq 0$

$$
\left\|h_{j+1}\right\|_{C^{1}(\bar{\Omega})} \leq \varepsilon_{j+1} \leq \frac{1}{4} \tilde{\nu}\left(S_{j}, \varepsilon_{j}\right) \leq \frac{1}{4} \varepsilon_{j} \leq \frac{\varepsilon}{4^{j}} .
$$

Obviously, the series $\sum_{j=0}^{\infty} h_{j}$ converges in $C^{1}$-norm to a limit $h_{\infty}$, and $\left\|h_{\infty}-h_{0}\right\|_{C^{1}(\bar{\Omega})} \leq \min \left\{\varepsilon, \delta\left(S_{1}\right) / 2\right\}$. Moreover, for any $j$,

$$
\left\|h_{\infty}-\sum_{i=0}^{j} h_{i}\right\|_{C^{0}(\bar{\Omega})} \leq \frac{1}{4} \tilde{\nu}\left(S_{j}, \varepsilon_{j}\right)+\frac{1}{3} \varepsilon_{j+1} \leq \frac{1}{3} \tilde{\nu}\left(S_{j}, \varepsilon_{j}\right) .
$$

Hence the number $N_{h_{\infty}}\left(S_{j}\right)$ of $C^{2+\alpha}$-smooth solutions $u$ of the problem

$$
\left\{\begin{array}{l}
-\Delta u+f(u, x)=h_{\infty}(x) \text { in } \Omega, \\
\left.u\right|_{\partial \Omega}=0,
\end{array}\right.
$$

such that $\|u\|_{C^{0}(\bar{\Omega})} \leq S_{j}$ satisfies the inequality

$$
\begin{aligned}
\ln N_{h_{\infty}}\left(S_{j}\right) \leq \overline{\boldsymbol{\Theta}}_{1}(N, \operatorname{diam} \Omega)( & (\operatorname{dil} \Omega)^{N} S_{j}^{N}\left(\bar{M}_{0}\left(S_{j}\right)+\left\|h_{0}\right\|_{C^{0}(\bar{\Omega})}\right)^{N} \\
& \left.\times\left(\bar{M}_{2}\left(S_{j}\right)\right)^{7 N^{2} / 4+N}+\bar{M}_{2}\left(S_{j}\right)^{N} \ln 1 / \varepsilon_{j}\right),
\end{aligned}
$$

for an appropriate constant $\bar{\Theta}_{1}(N, \operatorname{diam} \Omega)$.

Now let us choose a sequence $\left\{S_{j}\right\}_{j=1}^{\infty}$ to make (1.9) follow from (5.4). Note that for $j \geq 1$,

$$
\begin{aligned}
\ln \frac{1}{\varepsilon_{j+1}} \leq & C_{19}(N, \operatorname{diam} \Omega) \\
& \times \max \left\{( \operatorname { d i l } \Omega ) ^ { N } S _ { j } ^ { N } \left(\bar{M}_{0}\left(S_{j}\right)+\right.\right. \\
& \left.\left\|h_{0}\right\|_{C^{0}(\bar{\Omega})}\right)^{N} \bar{M}_{2}\left(S_{j}\right)^{7 N^{2} / 4+N / 2} \\
& \left.+\bar{M}_{2}\left(S_{j}\right)^{N / 2} \ln \frac{1}{\varepsilon_{j}}, \ln \frac{1}{\delta\left(S_{j+1}\right)}\right\},
\end{aligned}
$$

for some constant $C_{19}(N, \operatorname{diam} \Omega$ ) (depending only on $N$ and $\operatorname{diam} \Omega$ ). But using (3.48) we see that for some $C_{20}(N$, diam $\Omega)$,

$$
\ln \left(1 / \delta\left(S_{j+1}\right)\right) \leq C_{20}(N, \operatorname{diam} \Omega) \ln \bar{M}_{2}\left(S_{j+1}\right) \text {. }
$$

Hence for some constant $C_{21}(N, \operatorname{diam} \Omega)$,

$$
\begin{aligned}
\ln \frac{1}{\varepsilon_{j+1}} \leq & C_{21}(N, \operatorname{diam} \Omega) \\
& \times\left((\operatorname{dil} \Omega)^{N} S_{j}^{N}\left(\bar{M}_{0}\left(S_{j}\right)+\left\|h_{0}\right\|_{C^{0}(\bar{\Omega})}\right)^{N} \bar{M}_{2}\left(S_{j}\right)^{7 N^{2} / 4+N / 2}\right. \\
& \left.+\bar{M}_{2}\left(S_{j}\right)^{N / 2} \ln \frac{1}{\varepsilon_{j}}+\ln \bar{M}_{2}\left(S_{j+1}\right)\right) .
\end{aligned}
$$


We recall that by the conditions of Theorem $1.2 \lim _{S \rightarrow+\infty} \bar{M}_{2}(S)=+\infty$. It is obvious now that one can choose $S_{j+1}$ large enough to ensure that

$$
\begin{aligned}
2\left((\operatorname{dil} \Omega)^{N} S_{j+1}^{N}\left(\bar{M}_{0}\left(S_{j+1}\right)+\left\|h_{0}\right\|_{C^{0}(\bar{\Omega})}\right)^{N}\right. \\
\left.\quad \times \bar{M}_{2}\left(S_{j+1}\right)^{7 N^{2} / 4+N}+\bar{M}_{2}\left(S_{j+1}\right)^{N+\delta} \ln 1 / \varepsilon\right) \\
\geq(\operatorname{dil} \Omega)^{N} S_{j+1}^{N}\left(\bar{M}_{0}\left(S_{j+1}\right)+\left\|h_{0}\right\|_{C^{0}(\bar{\Omega})}\right)^{N} \\
\quad \times \bar{M}_{2}\left(S_{j+1}\right)^{7 N^{2} / 4+N}+\bar{M}_{2}\left(S_{j+1}\right)^{N} \ln 1 / \varepsilon_{j+1} .
\end{aligned}
$$

This completes the proof of Theorem 1.2.

\section{ACKNOWLEDGMENTS}

The main idea of this work is to use Yomdin's quantitative version of Sard's theorem + relative compactness of the set of solutions in order to obtain estimates on the number of solutions of a generic boundary value problem (1.3). This idea was suggested to me by Professor Y. Yomdin. I would like to express my gratitude to Professor Yomdin for this crucial suggestion and also for the encouragement without which this paper would have never been written.

I would like to thank Professor L. Nirenberg and Professor H. Brezis for very valuable comments and suggestions.

\section{REFERENCES}

1. A. Bahri and $\mathrm{H}$. Berestycki, A perturbation method in critical point theory and applications, Trans. Amer. Math. Soc. 267 (1981), 1-32.

2. C. Bandle, Isoperimetric inequalities and applications, Pitman, New York, 1980.

3. P. Bérard, From vanishing theorems to estimating theorems: the Bochner techniques revisited, Bull. Amer. Math. Soc. 19 (1988), 371-406.

4. H. Ehrmann, Über die Existenz der Lösungen von Randwertaufgaben bei gewöhnilcher nichtlinearen Differentialgleichungen zweiter Ordnung, Math. Ann. 134 (1957), 167-194.

5. C. Foiaş and R. Temam, A generic property of the set of stationary solutions of Navier-Stokes equations, Turbulence and Navier-Stokes Equations, Proc. Conf. (University of Paris-Sud, Orsay, June 12-13, 1975 (R. Teman, ed.), Lecture Notes in Math., vol. 565, Springer-Verlag, Berlin and New York, 1976.

6. _ Structure of the set of stationary solutions of the Navier-Stokes equations, Comm. Pure Appl. Math. 30 (1977), 149-164.

7. S. Fučik and V. Lovicar, Periodic solutions of the equation $x^{\prime \prime}(t)+g(x(t))=p(t)$, Časopis Pěst. Mat. 100 (1975), 160-175.

8. S. Fučik and A. Kufner, Nonlinear differential equations, Elsevier, Amsterdam, 1980.

9. A. N. Kolmogorov and V. M. Tihomirov, e-enthropy and e-capacity of sets in functional spaces, Amer. Math. Soc. Transl. (2) 17 (1961), 277-364.

10. L. Nirenberg, Topics in nonlinear functional analysis, New York, Univ., 1974.

11. B. Ruf and S. Solimini, On a class of superlinear Sturm-Liouville problems with arbitrarily many solutions, SIAM J. Math. Anal. 17 (1986), 761-771.

12. J. C. Saut and R. Temam, Generic properties of nonlinear boundary value problems, Comm. Partial Differential Equations 4 (1979), 293-319.

13. S. Smale, An infinite dimensional version of Sard's Theorem, Amer. J. Math. 87 (1965), 861-866.

14. Y. Yomdin, The geometry of critical and near critical values of differentiable mapping, Math. Ann. 264 (1983), 495-515. 
15. __ Metric semialgebraic geometry with applications in smooth analysis (to appear).

16. __ Global bounds for the Betti numbers of regular fibers of differentiable mappings, Topology 24 (1985), 145-152.

17. A. Ambrosetti and G. Prodi, On the inversion of some differentiable mappings with singularities between Banach spaces, Ann. of Math. 93 (1972), 231-246.

18. M. S. Berger and P. T. Church, Folds and cusps in Banach spaces, with applications to nonlinear partial differential equations. I, Indiana Univ. Math. J. 34 (1985), 1-19.

19. __ Folds and cusps in Banach spaces, with applications to nonlinear partial differential equations. II, Trans. Amer. Math. Soc. 307 (1988), 225-244.

20. M. S. Berger and E. Podolak, On the solution of a nonlinear Dirichlet problem, Indiana Univ. Math. J. 24 (1974-75), 837-846.

21. K. Leichtweiss, Konvexe Mengen, VEB Deutscher Verlag der Wissenschaften, Berlin, 1980.

22. H. P. McKean and J. C. Scovel, Geometry of some simple nonlinear differential operators, Ann. Scuola Norm. Sup. Pisa Cl. Sci. (4) 13 (1986), 299-346.

23. P. H. Rabinowitz, A survey of some superlinear problems, Nonlinear Diffusion Equations and their Equilibrium States. II (W.-M. Ni, L. A. Peletier, and J. Serrin eds.), SpringerVerlag, Berlin and New York, 1988, pp. 217-233.

24. M. Struwe, Infinitely many critical points for functionals which are not even and applications to superlinear boundary value problems, Manuscripta Math. 32 (1980), 335-364.

Department of Theoretical Mathematics, Weizmann Institute of Science, P.O.B. 26, REHOVOT 76100, ISRAEL

Current address: Department of Mathematics, University of Toronto, Toronto, Ontario M5S 1A1, Canada 